\title{
Far-infrared study of tracers of oxygen chemistry in diffuse clouds
}

\author{
H. Wiesemeyer ${ }^{1}$, R. Güsten ${ }^{1}$, S. Heyminck ${ }^{1}$, H.W. Hübers ${ }^{2,3}$, K.M. Menten ${ }^{1}$, D.A. Neufeld ${ }^{4}$, H. Richter ${ }^{2}$, \\ R. Simon ${ }^{5}$, J. Stutzki ${ }^{5}$, B. Winkel ${ }^{1}$, and F. Wyrowski ${ }^{1}$
}

\author{
1 Max-Planck-Institut für Radioastronomie, Auf dem Hügel 69, 53121 Bonn, Germany \\ e-mail: hwiese@mpifr.de \\ 2 Deutsches Zentrum für Luft- und Raumfahrt, Institute of Optical Sensor Systems, Rutherfordstr. 2, 12489 Berlin, Germany \\ 3 Humboldt-Universität zu Berlin, Department of Physics, Newtonstr. 15, 12489 Berlin, Germany \\ 4 The Johns Hopkins University, 3400 North Charles St. Baltimore, MD 21218, USA \\ 5 I. Physikalisches Institut, Universität zu Köln, Zülpicher Str. 77, 50937 Köln, Germany
}

Received 6 May 2015 / Accepted 3 October 2015

\begin{abstract}
Context. The chemistry of the diffuse interstellar medium rests upon three pillars: exothermic ion-neutral reactions ("cold chemistry"), endothermic neutral-neutral reactions with significant activation barriers ("warm chemistry"), and reactions on the surfaces of dust grains. While warm chemistry becomes important in the shocks associated with turbulent dissipation regions, the main path for the formation of interstellar $\mathrm{OH}$ and $\mathrm{H}_{2} \mathrm{O}$ is that of cold chemistry.

Aims. The aim of this study is to observationally confirm the association of atomic oxygen with both atomic and molecular gas phases, and to understand the measured abundances of $\mathrm{OH}$ and $\mathrm{OH}^{+}$as a function of the available reservoir of $\mathrm{H}_{2}$.

Methods. We obtained absorption spectra of the ground states of $\mathrm{OH}, \mathrm{OH}^{+}$and $\mathrm{O}$ I with high-velocity resolution, with GREAT onboard SOFIA, and with the THz receiver at the APEX. We analyzed them along with ancillary spectra of HF and CH from HIFI. To deconvolve them from the hyperfine structure and to separate the blend that is due to various velocity components on the sightline, we fit model spectra consisting of an appropriate number of Gaussian profiles using a method combining simulated annealing with downhill simplex minimization. Together with $\mathrm{HF}$ and/or $\mathrm{CH}$ as a surrogate for $\mathrm{H}_{2}$, and $\mathrm{HI} \lambda 21 \mathrm{~cm}$ data, the molecular hydrogen fraction $f_{\mathrm{H}_{2}}^{\mathrm{N}}=N\left(\mathrm{H}_{2}\right) /\left(N(\mathrm{H})+2 N\left(\mathrm{H}_{2}\right)\right)$ can be determined. We then investigated abundance ratios as a function of $f_{\mathrm{H}_{2}}^{\mathrm{N}}$.

Results. The column density of $\mathrm{O}$ I is correlated at a high significance with the amount of available molecular and atomic hydrogen, with an atomic oxygen abundance of $3 \times 10^{-4}$ relative to $\mathrm{H}$ nuclei. While the velocities of the absorption features of $\mathrm{OH}_{\text {and }} \mathrm{OH}^{+}$are loosely correlated and reflect the spiral arm crossings on the sightline, upon closer inspection they display an anticorrespondence. The arm-to-interarm density contrast is found to be higher in $\mathrm{OH}$ than in $\mathrm{OH}^{+}$. While both species can coexist, with a higher abundance in $\mathrm{OH}$ than in $\mathrm{OH}^{+}$, the latter is found less frequently in absence of $\mathrm{OH}$ than the other way around, which is a direct consequence of the rapid destruction of $\mathrm{OH}^{+}$by dissociative recombination when not enough $\mathrm{H}_{2}$ is available. This conjecture has been substantiated by a comparison between the $\mathrm{OH} / \mathrm{OH}^{+}$ratio with $f_{\mathrm{H}_{2}}^{\mathrm{N}}$, showing a clear correlation. The hydrogen abstraction reaction chain $\mathrm{OH}^{+}\left(\mathrm{H}_{2}, \mathrm{H}\right) \mathrm{H}_{2} \mathrm{O}^{+}\left(\mathrm{H}_{2}, \mathrm{H}\right) \mathrm{H}_{3} \mathrm{O}^{+}$is confirmed as the pathway for the production of $\mathrm{OH}$ and $\mathrm{H}_{2} \mathrm{O}$. Our estimate of the branching ratio of the dissociative recombination of $\mathrm{H}_{3} \mathrm{O}^{+}$to $\mathrm{OH}$ and $\mathrm{H}_{2} \mathrm{O}$ is confined within the interval of 84 to $91 \%$, which matches laboratory measurements (74 to 83\%). - A correlation between the linewidths and column densities of $\mathrm{OH}^{+}$features is found to be significant with a false-alarm probability below $5 \%$. Such a correlation is predicted by models of interstellar MHD turbulence. For $\mathrm{OH}$ the same correlation is found to be insignificant because there are more narrow absorption features.

Conclusions. While it is difficult to assess the contributions of warm neutral-neutral chemistry to the observed abundances, it seems fair to conclude that the predictions of cold ion-neutral chemistry match the abundance patterns we observed.
\end{abstract}

Key words. ISM: abundances - ISM: atoms - ISM: clouds - ISM: lines and bands - ISM: molecules - ISM: structure

\section{Introduction}

The gas in the diffuse atomic and molecular phases of the interstellar medium (ISM) contributes roughly $5 \%$ to the total visible mass of our Galaxy (Sodroski et al. 1997; Kafle et al. 2014) and represents the reservoir out of which the denser gas phases are formed when crossing the spiral arms and the Galactic bulge. While most of the molecular gas is concentrated in molecular clouds and their star-forming cores, the discovery of molecules in the diffuse phase (Dunham 1937; Swings \& Rosenfeld 1937) came as a surprise, given the strong dissociating UV radiation in the Galaxy. An account of the historical development is given in
Cox (2005). Because diffuse clouds can be observed in spectroscopic tracers from UV to radio wavelengths, the diffuse gas was soon shown to be chemically extremely rich (Snow \& McCall 2006, further references therein). The chemistry of diffuse clouds has been reviewed by van Dishoeck (1998). The accepted theory rests on three pillars: cold chemistry driven by ion-neutral reactions, warm chemistry whose endothermic reactions are powered by the dissipation of supersonic turbulence, and chemistry on surfaces of dust grains acting as catalyst.

While the first comprehensive models of chemistry in diffuse gas considered a realistic cloud structure and radiative transfer (Heck et al. 1992; van Dishoeck \& Black 1986) and thereby 
Table 1. Studied species and transitions.

\begin{tabular}{|c|c|c|c|c|c|c|c|}
\hline Species & Transition & $\begin{array}{l}\text { Hyperfine } \\
\text { component }\end{array}$ & $\begin{array}{c}\text { Frequency } \\
{[\mathrm{GHz}]}\end{array}$ & $\begin{array}{c}\Delta v_{\mathrm{HFS}} \\
{\left[\mathrm{km} \mathrm{s}^{-1}\right]}\end{array}$ & $\begin{array}{c}A_{\mathrm{E}} \\
{\left[\mathrm{s}^{-1}\right]}\end{array}$ & $\begin{array}{l}E_{\mathrm{U}} \\
{[\mathrm{K}]}\end{array}$ & Origin \\
\hline OI & ${ }^{3} \mathrm{P}_{1} \leftarrow{ }^{3} \mathrm{P}_{2}$ & & 4744.7775 & & $8.91 \times 10^{-5}$ & 227.76 & GREAT \\
\hline \multirow{3}{*}{$\mathrm{OH}$} & ${ }^{2} \Pi_{3 / 2} J=5 / 2 \leftarrow 3 / 2$ & $F=2^{-} \leftarrow 2^{+}$ & 2514.2987 & +2.1 & 0.0137 & 120.75 & GREAT \\
\hline & & $F=3^{-} \leftarrow 2^{+}$ & 2514.3167 & 0.0 & 0.1368 & & \\
\hline & & $F=2^{-} \leftarrow 1^{+}$ & 2514.3532 & -4.4 & 0.1231 & & \\
\hline $\mathrm{HF}$ & $J=1 \leftarrow 0$ & & 1232.4763 & & 0.0242 & 59.15 & HIFI \\
\hline \multirow[t]{7}{*}{$\mathrm{OH}^{+}$} & $N=1 \leftarrow 0, J=1 \leftarrow 1$ & $F=1 / 2 \leftarrow 1 / 2$ & 1032.9979 & +35.0 & 0.0141 & 49.58 & APEX \\
\hline & & $F=3 / 2 \leftarrow 1 / 2$ & 1033.0044 & +33.1 & 0.0035 & & \\
\hline & & $F=1 / 2 \leftarrow 3 / 2$ & 1033.1118 & +2.0 & 0.0070 & & \\
\hline & & $F=3 / 2 \leftarrow 3 / 2$ & 1033.1186 & 0.0 & 0.0176 & & \\
\hline & $N=1 \leftarrow 0, J=2 \leftarrow 1$ & $F=5 / 2 \leftarrow 3 / 2$ & 971.8038 & 0.0 & 0.0182 & 46.64 & HIFI \\
\hline & & $F=3 / 2 \leftarrow 1 / 2$ & 971.8053 & -0.46 & 0.0152 & & \\
\hline & & $F=3 / 2 \leftarrow 3 / 2$ & 971.9192 & -35.6 & 0.0030 & & \\
\hline \multirow[t]{3}{*}{$\mathrm{CH}$} & $N=1, J=3 / 2 \leftarrow 1 / 2$ & $F=2^{-} \leftarrow 1^{+}$ & 536.7611 & 19.3 & 0.0006 & 25.76 & HIFI \\
\hline & & $F=1^{-} \leftarrow 1^{+}$ & 536.7819 & 7.7 & 0.0002 & & \\
\hline & & $F=1^{-} \leftarrow 0^{+}$ & 536.7957 & 0.0 & 0.0004 & & \\
\hline
\end{tabular}

Notes. Frequencies, Einstein coefficients, and upper level energies are from the JPL (Pickett et al. 1998) and CDMS (Müller et al. 2001) databases.

corrected the previously underestimated self-shielding against the interstellar UV radiation field, they considered mainly cold chemistry and accounted for grain surface chemistry only in the formation of molecular hydrogen. Warm chemistry has been studied by Godard et al. (2009), making use of our best knowledge of interstellar turbulence and its dissipation. Vasyunin et al. (2009) have demonstrated the Monte Carlo treatment of gasgrain chemistry in large reaction networks.

The advent of low-noise Terahertz receivers with highresolution spectrometers such as HIFI (de Graauw et al. 2010) and GREAT ${ }^{1}$ (Heyminck et al. 2012) onboard the Herschel and SOFIA observatories, respectively, offered a new opportunity to derive column densities of light hydrides from measurements of absorption out of the ground state. Their precision is only limited by the radiometric noise and the accuracy of the calibration of the illuminating continuum radiation, but not fraught anymore with uncertain assumptions regarding the excitation of the radio lines used previously.

All atomic and molecular constituents of the network of chemical reactions leading to the formation of interstellar water and hydroxyl are accessible to observations. The latest contribution to these studies is the high-resolution spectroscopy of the ground-state transition of the fine structure of atomic oxygen (OI) with GREAT. Because of its usefulness as a tracer for the total gas mass in diffuse clouds, it allows us to understand the abundance of interstellar water and hydroxyl in more detail than previously possible. The latter two species are both mainly formed from the $\mathrm{OH}^{+}$cation, whose abundance depends on the reservoir of available hydrogen and its molecular fraction, on the cosmic ray ionization rate, and on the initially available O I. The $\mathrm{OH}^{+}$cation was first detected by Wyrowski et al. (2010). Motivated by the increasing amount of high-quality data, models, and databases for chemical networks (e.g., OSU ${ }^{2}$, and $\mathrm{KIDA}^{3}$, Wakelam et al. 2012), we investigate the abundance patterns in the diffuse gas of the spiral arms on the sightlines

\footnotetext{
GREAT is a development by the MPI für Radioastronomie and the KOSMA/ Universität zu Köln, in cooperation with the MPI für Sonnensystemforschung and the DLR Institut für Planetenforschung. 2 http://www.physics.ohio-state.edu/ eric/research. html

3 http://kida.obs.u-bordeaux1.fr/
}

to continuum sources in the first and fourth quadrants of the Galaxy.

The plan of this work is as follows: Sect. 2 presents the technical aspects of the observations and the data analysis. Section 3 provides a digest of the data used for this study. A phenomenological description of the results can be found in Appendix A. The discussion in Sect. 4 presents a deeper analysis of the data, referring to Appendix B for a note on averaging abundances. We conclude the paper with Sect. 5. The most important chemical reactions addressed in this work are summarized in Appendix C; a few simple chemical models are presented in Appendix D. In Appendix E we assess the reliability of the $\mathrm{CH}$ and $\mathrm{HF}$ groundstate transitions as tracers for $\mathrm{H}_{2}$. A comparison of hydroxyl and oxygen spectroscopy with PACS (Poglitsch et al. 2010) and GREAT is shown in Appendix F to demonstrate the crosscalibration of the two instruments.

\section{Observations and data analysis}

The following sections provide an overview of the technical aspects of our observations with GREAT and with the terahertz receiver at the $\mathrm{APEX}^{4}$ (Leinz et al. 2010). Our source selection consists of sources from the Herschel guaranteed time program PRISMAS (P.I. M. Gerin ${ }^{5}$ ) and far-IR-bright hot cores in the fourth quadrant of the Galaxy (from ATLASGAL, Csengeri et al. 2014). The set of data that we obtained was completed with HIFI spectra from the Herschel Science Archive; the underlying observations are described elsewhere (e.g., Gerin et al. 2010, further references therein). Table 1 summarizes the observed transitions and the origin of the data.

\subsection{Terahertz spectroscopy of $\mathrm{OH}$ and OI with GREAT}

We conducted the spectroscopy of the ${ }^{2} \Pi_{3 / 2} J=5 / 2 \leftarrow 3 / 2$ ground-state line and of the excited ${ }^{2} \Pi_{1 / 2} J=5 / 2 \leftarrow 3 / 2$ line

4 This publication is based on data acquired with the Atacama Pathfinder Experiment (APEX). APEX is a collaboration between the Max-Planck-Institut für Radioastronomie, the European Southern Observatory, and the Onsala Space Observatory.

5 http://astro.ens.fr/?PRISMAS 
of OH with the $M_{a}$ channel of GREAT onboard SOFIA. To remove instrumental and atmospheric fluctuations and to suppress standing waves in the spectra, the double beam-switch mode was used, chopping with the secondary mirror at a rate of $1 \mathrm{~Hz}$ and with an amplitude of typically $60^{\prime \prime}$. The new data, mainly from the fourth quadrant, were obtained on the southern deployment flights from New Zealand in July 2013, as a part of the observatory's cycle 1 . Pointing and tracking were accurate to better than $2^{\prime \prime}$. The sightlines toward G10.47 and W31C were observed in April 2013, on northern hemisphere flights of the same cycle. W49N, W51e2 and G34.26 were already observed in 2012 on the basic science flights (Wiesemeyer et al. 2012). With the successful commissioning of GREAT's H channel in May 2014, the fine-structure line of atomic oxygen became accessible for spectrally resolved studies. A novel NbN HEB waveguide mixer (Büchel et al. 2015) was pumped by a quantum cascade laser as local oscillator (Richter et al. 2015); the Doppler correction was applied offline.

The southern deployment flights offered excellent observing conditions. Typical system temperatures range between 6340 and $8300 \mathrm{~K}$ (single sideband scale), with a median system temperature of $6500 \mathrm{~K}$, for water vapor columns between 4.3 and $14.2 \mu \mathrm{m}$, with a median of $10.9 \mu \mathrm{m}$ water vapor. The weather conditions on the northern flights in April 2013 were slightly less favorable but still very good, with system temperatures and water vapor columns of $6640 \mathrm{~K}$ and $15 \mu \mathrm{m}$, respectively (median values). In May 2014 the oxygen line was observed at a system temperature of $2400 \mathrm{~K}$, with a water vapor column of $6.6 \mu \mathrm{m}$ (median values). The calibration of the $\mathrm{H}$-channel and in the low-frequency $\mathrm{L}_{2}$ channel is consistent and yielded the same water vapor column.

In 2013 the main-beam efficiencies were significantly improved thanks to new optics and a smaller scatter cone. They were measured by means of total power cross-scans on Jupiter in April 2013, yielding a value of $\eta_{\mathrm{mb}}=0.70$ for the $M_{a}$ channel. The major and minor sizes of the Jupiter disk were 34.5 and 32 '. 2 , respectively. For the southern hemisphere campaign we observed Saturn as calibrator, confirming this efficiency. The beam width (FWHM) is $12^{\prime \prime}$. For the H-channel, the main beam efficiency is 0.66 , with a 6.' 6 beam width. These values were determined on Mars, which had a diameter of $13^{\prime \prime}$.

For the spectral analysis of our data we used the XFFTS spectrometer (Klein et al. 2012). The spectral resolution of $76.3 \mathrm{kHz}$ was smoothed to a velocity resolution of $0.36 \mathrm{~km} \mathrm{~s}^{-1}$ (in a few cases $0.72 \mathrm{~km} \mathrm{~s}^{-1}$ ).

\section{2. $\mathrm{OH}^{+}$spectroscopy}

The ground-state line of $\mathrm{OH}^{+}$was observed in July 2010 at the APEX telescope with the MPIfR dual-channel terahertz receiver (Leinz et al. 2010), with the exception of G34.26, W49N and W51e2, whose $\mathrm{OH}^{+}$HIFI spectra were retrieved from the Herschel Science Archive. Details of the instrumental set-up at the APEX are given in Qiu et al. (2011).

The weather conditions under which we obtained the $\mathrm{THz}$ data confirm the excellence of the site, with the water vapor columns ranging from 0.15 to $0.25 \mathrm{~mm}$, resulting in a median single-sideband system temperature of $1280 \mathrm{~K}$. The telescope pointing was conducted on the strong dust continuum of the background sources. The data were taken in wobbler-switching mode. The fast Fourier transform (FFT) spectrometers delivered data with channel spacings of $76.3 \mathrm{kHz}$, smoothed to a velocity resolution of $1.7 \mathrm{~km} \mathrm{~s}^{-1}$.

\subsection{Data reduction}

The data were calibrated with standard methods: Calibration loads at ambient and liquid nitrogen temperature were measured approximately every 10 min to derive the count-rate to Kelvin conversion, allowing us to measure the brightness temperature of the $\mathrm{sky}^{6}$; the atmosphere model reproducing the latter was then used for the opacity correction. Details are described in Guan et al. (2012) and Hafok \& Polehampton (2013) for the calibration of data from GREAT and from the APEX, respectively. For the aims of this work, that is, for absorption spectroscopy in front of a continuum background source, the degree of accuracy of the absolute calibration scale would be inconsequential for a single-sideband receiving system. However, because of the double-sideband reception, an adequate correction for the different atmospheric transmission in both sidebands is needed. For the $\mathrm{OH}^{+}$lines, which are observed in the upper sideband, the ratio of the signal-to-image band transmission is close to unity for a sightline to the zenith under the weather conditions mentioned above, and for our observations it has a median value of 0.4. Thanks to the good observing conditions, the ratio between the transmissions in the signal- to image-band were close to unity for GREAT.

The effect of the continuum calibration on the deduced column densities is discussed in the next paragraph. We provide a comparison with the continuum calibration of PACS in Appendix F.

\subsection{Data analysis}

The power of ground-state absorption spectroscopy, namely, the direct determination of column densities free from uncertain assumptions, has been demonstrated in a number of publications (for $\mathrm{OH}$, Wiesemeyer et al. 2012). For a Gaussian line profile, the opacity of an absorption line with several velocity and hyperfine components can be decomposed and reads, for a velocity component $i$ and a hyperfine component $j$,

$\tau_{i j, v}=\sqrt{\frac{\ln 2}{\pi}} \frac{A_{\mathrm{E}, j} c^{3}}{4 \pi \Delta v_{i} v_{j}^{3}} \frac{g_{\mathrm{u}, j}}{g_{l, j}} N w_{j} \exp \left(-4 \ln 2\left(\frac{v-v_{0, i j}}{\Delta v_{i}}\right)^{2}\right)$,

with the following quantities: the Einstein coefficient of the transition, $A_{\mathrm{E}, j}$, the full width at half maximum of the Gaussian line profile, $\Delta v_{i}$, the degeneracies of the upper and lower levels involved in the transition, $g_{\mathrm{u}, j}$ and $g_{l, j}$, the total column density of the absorbing molecular or atomic species, $N$, and the center velocity of the velocity component, $v_{0, i j}$. The velocity scale of the obtained spectra typically refers to the rest frequency of the strongest hyperfine component in the local standard of rest; therefore $v_{0, i j}$ depends on the hyperfine component (the corresponding velocity offsets are listed in Table 1). The factor $w_{j}$ accounts for the fraction of species that are in the corresponding ground state. For the physical conditions prevailing in diffuse (atomic and molecular) and translucent clouds, that is, for gas temperatures ranging from 15 to $100 \mathrm{~K}$, densities from $\sim 10$ to $\sim 10^{3} \mathrm{~cm}^{-3}$ and extinctions from $A_{\mathrm{V}}=0-2$ (Snow \& McCall 2006), the ground-state occupation can be approximated by thermal equilibrium at the temperature $T_{\mathrm{cmb}}$ of the cosmic microwave background (CMB), that is,

$w_{j}=g_{j} / Q\left(T_{\mathrm{cmb}}\right)$,

6 Throughout this paper, all temperatures are Rayleigh-Jeans equivalent temperatures, unless stated otherwise. 
where $Q$ is the partition function. We assess the validity of this assumption in Sect. 2.5 and conclude this paragraph with numerical details regarding our ansatz for the modeling, and the error analysis.

As a result of the blend of velocity components and hyperfine components, the data analysis is not straightforward. The hyperfine structure can either be deconvolved (Godard et al. 2012) or direcly fitted to the data. Here we used the second option because deconvolution algorithms tend to produce spurious features unless the spectral baselines are excellent, which is not the case for some data analyzed here. Owing to the large number of free parameters, fitting many velocity components, accounting for hyperfine blends, is not an easy task either, however. The XCLASS code ${ }^{7}$, originally created for analyzing line surveys (e.g., Comito et al. 2005), is widely used for this purpose and is based on the Levenberg-Marquardt algorithm (e.g., Press et al. 1992). An alternative method, used here, is the extension of discrete minimization by simulated annealing (Metropolis et al. 1953) to continuous, nonlinear minimization problems with a large number of free parameters. The basic idea (Press et al. 1992) is to combine the Metropolis algorithm with the downhill simplex method. To avoid an undesired convergence to a local minimum, uphill steps are sometimes accepted, with a probability defined by a "temperature" that is gradually lowered, in analogy to the thermodynamic description of annealing. Both approaches have their strengths and weaknesses. While their extensive discussion is beyond the scope of this paper, we conclude that simulated annealing leads to satisfactory results once an efficient annealing scheme (i.e., variation of the temperature along a sequence of iterations) is found. We also fit line profiles for species that do not display hyperfine structure (HF, oxygen), for two reasons: First, we obtain a description of the underlying velocity profiles, which are otherwise difficult to identify because the components blend with each other, and, second, the granularity in the obtained column density profiles is avoided, while the $\chi^{2}$ function of the fit allows estimating the errors.

The main-beam brightness temperature (Rayleigh-Jeans scale) of the observed spectrum is

$T_{\mathrm{RJ}}(v)=T_{\mathrm{RJ}, \mathrm{c}}^{(\mathrm{img})}+T_{\mathrm{RJ}, \mathrm{c}}^{(\mathrm{sig})} \exp \left(-\sum_{i=1}^{N_{\mathrm{vc}}} \sum_{j=1}^{N_{\mathrm{hfc}}} \tau_{i j, v}\right)$,

where $T_{\mathrm{RJ}, \mathrm{c}}^{(\mathrm{sig})}$ and $T_{\mathrm{RJ}, \mathrm{c}}^{(\mathrm{img})}$ are the Rayleigh-Jeans temperatures of the continuum in the signal and image band, and $N_{\mathrm{vc}}$ and $N_{\mathrm{hfc}}$ are the number of velocity components and hyperfine components, respectively. Uncertainties in the determination of the signal band calibration temperature cancel out because they equally affect the line and continuum temperature. The continuum level may also be affected (1) by the accuracy of the image band calibration and (2) by standing waves whose power adds to that of the continuum. As mentioned above, case (1) could arise from a slope between the atmospheric transmission in the signal and image band. It can easily be shown that the resulting uncertainty in the opacity of the absorption line is (all temperatures and noise levels are on the Rayleigh-Jeans scale, the subscript RJ is therefore omitted)

$\sigma_{\tau}=\sqrt{\sigma_{T_{\mathrm{c}}}^{2}+\left[\sigma_{\mathrm{r}} T_{\mathrm{c}}^{(\mathrm{sig})}\right]^{2}} \frac{\mathrm{e}^{\tau}}{T_{\mathrm{c}}^{(\mathrm{sig})}}$,

where $\sigma_{\mathrm{r}}^{2}$ is the variance of ratio of the image band to the signal band continuum. For the purpose of a rough error estimate we

\footnotetext{
7 https://www . astro.uni-koeln.de/projects/schilke/ XCLASS
}

set $\sigma_{\mathrm{r}}=0.1$ as a conservative assumption, and $T_{\mathrm{c}}^{(\mathrm{sig})} \mathrm{e}^{-\tau}$ to the observed line temperature $T_{\mathrm{L}}(=0 \mathrm{~K}$ for saturated absorption, and $=T_{\mathrm{c}}^{(\mathrm{sig})}$ out of the line). As expected, $\sigma_{\tau} \rightarrow \infty$ for a saturated line. As a bona fide estimate of $\sigma_{T_{\mathrm{c}}}^{2}$ we can use the $\chi^{2}$ of the fit. Another approach, used in Wiesemeyer et al. (2012), consists of adding random noise to the fits to the absorption line systems, and to determine the line parameters of these fits. The distribution of fit parameters among a significant number of simulated spectra yields empirical standard deviations. The advantage of this method is that is does not require the errors to display a normal distribution. However, for this study this approach is impractical because there are so many targets, sightline components, and species observed and analyzed. We therefore used the error estimate outlined in this paragraph. In both approaches, the errors are underestimated if instrumental bandpass ripples are misinterpreted as weak but broad absorption features.

\subsection{OH level populations}

We finally examine our assumption that the level distribution can be described by a thermal equilibrium with the CMB. We limit the discussion to $\mathrm{OH}$, which is also an opportunity to illustrate the difficulties encountered when radio lines are used for abundance determinations. We apply two models (Table 3) that are thought to bracket the conditions in diffuse molecular and translucent clouds. The level population of $\mathrm{OH}$ was modeled with the Molpop-CPE code written by Elitzur \& Asensio Ramos (2006). Their idea is to extend the rate equations of escapeprobability methods with a sum of terms describing the radiative coupling of the level populations between the various zones of the underlying sheet- or slab-like geometries.

The results show that while in model 2 the populations of ${ }^{2} \Pi_{3 / 2}, J=3 / 2$ states of $\mathrm{OH}$ can still be well described by a local thermodynamic equilibrium (LTE) at the temperature of the cloud model, the assumption breaks down in model 1, with populations that are over-thermal by a factor 1.7. This implies that excitation studies employing only the radio lines of $\mathrm{OH}$ either describe the emitting gas inadequately or require non-LTE modeling. On the other hand, in both models the departure of the level populations from LTE at the temperature of the CMB $(2.73 \mathrm{~K})$ merely amounts to at most 1.024 , resulting in a negligible error in the determination of $\mathrm{OH}$ column densities (we show below that uncertainties in the calibration of the continuum level may lead to much larger errors).

A further study recalibrating the column densities of radio studies (e.g., Neufeld et al. 2002) with corrections deduced from far-infrared observations is highly desirable, but is beyond the scope of this paper and will be published separately. In Appendix D, we present simple demonstrations of the chemistry in these two cloud models.

\section{Results}

All spectra reveal complex absorption profiles, while O I, HF, and $\mathrm{CH}$ are also seen in emission at the velocities of the hot cores and their environment, defined by $\mathrm{OH}$ and $\mathrm{CH}_{3} \mathrm{OH}$ maser emission (Table 2). This also holds for the excited ${ }^{2} \Pi_{1 / 2}$, $J=3 / 2 \rightarrow 1 / 2$ line of $\mathrm{OH}$, often observed simultaneously with the $\mathrm{OH}$ spectra shown here (Csengeri et al. 2012). These data will be published separately: the chemistry in the embedded ultra-compact HII regions is very different from that of diffuse clouds and much more determined by endothermic reactions. Furthermore, the strength of absorption studies in ground-state 
Table 2. Continuum sources and observed species.

\begin{tabular}{|c|c|c|c|c|c|c|c|c|}
\hline & $T_{\mathrm{c}}{ }^{a}[\mathrm{~K}]$ & $\alpha(\mathrm{J} 2000)$ & $\delta(\mathrm{J} 2000)$ & $l$ & $b$ & \multicolumn{2}{|c|}{$v_{\mathrm{lsr}}\left[\mathrm{km} \mathrm{s}^{-1}\right]^{b}$} & Species \\
\hline G10.47 & 6.9 & 18:08:38.20 & $-19: 51: 50.0$ & $10^{\circ} 472$ & 0.027 & $(+48,+69)$ & $(+58,+77)$ & $\mathrm{CH}, \mathrm{OH}, \mathrm{OH}^{+}$ \\
\hline $\mathrm{G} 10.62^{(c)}$ & 10.4 & $18: 10: 28.69$ & $-19: 55: 50.0$ & 10.621 & -0.387 & $(-3,+1)$ & $(-10,+7)$ & $\mathrm{CH}, \mathrm{HF}, \mathrm{O}$, $\mathrm{OH}, \mathrm{OH}^{+}$ \\
\hline G34.26 & 9.0 & 18:53:18.70 & 01:14:58.0 & 34.257 & 0.154 & $(+55,+62)$ & $(+58,+62)$ & $\mathrm{HF}, \mathrm{OI}, \mathrm{OH}, \mathrm{OH}^{+}$ \\
\hline W49N & 12.3 & 19:10:13.20 & 09:06:12.0 & 43.166 & 0.012 & $(+2,+21)$ & $(-1,+19)$ & $\mathrm{CH}, \mathrm{HF}, \mathrm{OI}, \mathrm{OH}, \mathrm{OH}^{+}$ \\
\hline W51e2 & 8.0 & :43.90 & $14: 30: 30.5$ & 49.489 & -0.388 & $(+47,+72)$ & $(+50,+61)$ & $\mathrm{CH}, \mathrm{HF}, \mathrm{OH}, \mathrm{OH}^{+}$ \\
\hline G327.29 & 3.5 & $15: 53: 08.55$ & $-54: 37: 05.1$ & 327.294 & -0.580 & $(-72,-40)$ & $(-49,-37)$ & $\mathrm{CH}, \mathrm{OH}, \mathrm{OH}^{+}$ \\
\hline G330.95 & 10.4 & 16:09:53.01 & $-51: 54: 55.0$ & 330.954 & -0.182 & $(-102,-80)$ & $(-91,-87)$ & $\mathrm{CH}, \mathrm{OH}, \mathrm{OH}^{+}$ \\
\hline G332.83 & 7.1 & $16: 20: 10.65$ & $-50: 53: 17.6$ & 332.824 & -0.548 & $(-55,-53)$ & $(-59,-45)$ & $\mathrm{OH}, \mathrm{OH}^{+}$ \\
\hline G351.58 & 3.2 & $17: 25: 25.03$ & $-36: 12: 45.3$ & 351.581 & -0.353 & $(-101,-89)$ & $(-100,-87)$ & $\mathrm{OH}, \mathrm{OH}^{+}$ \\
\hline
\end{tabular}

Notes. ${ }^{(a)}$ Main-beam brightness temperature of continuum at $2.514 \mathrm{THz}$ (Rayleigh-Jeans scale). ${ }^{(b)}$ Columns 6 and 7 list the velocity ranges with $\mathrm{OH}$ and $\mathrm{CH}_{3} \mathrm{OH}$ maser emission, respectively. For the fourth quadrant, $\mathrm{OH}$ and $\mathrm{CH}_{3} \mathrm{OH}$ maser velocities are from Caswell (1998) and Caswell et al. (1995), for G34.26, from Fish et al. (2005) and Caswell et al. (1995), for W49N, from Deshpande et al. (2013) and Bartkiewicz et al. (2014), and for W51e2 from Fish et al. (2005) and Surcis et al. (2012), respectively. ${ }^{(c)}$ Also known as W31C.

Table 3. Conditions in diffuse cloud models and coefficients for departure from LTE of the population in $\mathrm{OH}^{2} \Pi_{3 / 2}, J=3 / 2$ levels.

\begin{tabular}{lcc}
\hline \hline & Model 1 & Model 2 \\
\hline & diffuse molecular & translucent \\
$\chi[$ Habing $]$ & 1.7 & 1.7 \\
$A_{\mathrm{V}}$ & 0.2 & 1 \\
$n_{\mathrm{H}}\left[\mathrm{cm}^{-3}\right]$ & 100 & 1000 \\
$f_{\mathrm{H}_{2}}^{\mathrm{n}}$ & 0.1 & 0.5 \\
$T_{\text {gas }}[\mathrm{K}]$ & 100 & 15 \\
$T_{\text {dust }}[\mathrm{K}]$ & 16 & 12 \\
\hline \multicolumn{3}{c}{ departure coefficients } \\
\hline$F=1-$ & 1.7580 & 0.9966 \\
$F=2-$ & 1.7565 & 0.9963 \\
$F=1+$ & 1.7398 & 0.9991 \\
$F=2+$ & 1.7384 & 0.9987 \\
\hline
\end{tabular}

Notes. The FUV field is parametrized in units of the Habing field (Draine field $=1.7$ Habing, Draine 1978). Defining quantities are from Snow \& McCall (2006) and from Voshchinnikov et al. (1999) for the dust temperature. The clouds are immersed in the interstellar radiation field as given by Mathis et al. (1983) and the cosmic microwave background. Departure coefficients are defined as the fractional level population with respect to the population for thermalization at the CMB temperature, $2.73 \mathrm{~K}$.

transitions - that is, column densities that can be directly inferred from observations - breaks down in the high-excitation environment of hot cores.

Our results are presented and discussed in view of the velocimetry and cartography of Galactic spiral arms, modeled by Vallée (2008) in an effort to provide a symbiosis of available $\mathrm{CO}$ and Hidata. These results were recently completed with the valuable distance measurements of Reid et al. (2014, further references therein, see also below) that we adopt in the following. For the hot cores in the fourth quadrant, fewer parallaxes are known. We use the distances of southern $6.7 \mathrm{GHz}$ methanol masers obtained by Green \& McClure-Griffiths (2011) from Galactic kinematics, removing the distance ambiguity with HIself-absorption, along with the distances determined by Wienen et al. (2015). We prefer sources in the Galactic plane, providing both strong continuum sources and allowing us to measure several spiral arm crossings in one spectrum, owing to the high resolution of GREAT. The available spectra for sightlines in the first quadrant of the Galaxy are shown in Fig. 1 and the corresponding column density profiles in Fig. 4.
The available spectra for sightlines in the forth quadrant of the Galaxy are shown in Fig. 2, with the corresponding column density profiles in Fig. 5. In the following $N_{\mathrm{v}}(Y)$ denotes the velocity-specific column density of species $Y$, that is, for a given velocity interval the area below the histogram for species $Y$ is the column density. We refer to Appendix A for comments on the individual sightlines in the first and fourth quadrants.

\section{Discussion}

For the following discussion we recall that all abundances and their ratios are derived from column densities, not from volume densities of individual cloud entities. By consequence, it proves difficult to distinguish different clouds on the sightline. Therefore our spectroscopy, although quantitative, cannot unambiguously identify the various phases of the diffuse gas on the sightline. However, owing to the high spectral resolution of GREAT, we can separate several spiral arm crossings, or identify interarm regions, by means of their characteristic velocity (Vallée 2008). The deduced column densities and abundances within these regions are summarized in Tables 4 and 5. Abundances were determined from the average ratio of column densities across a given velocity interval, and not from the ratio of averaged column densities. The rationale behind this is detailed in Appendix B. We use $\mathrm{HF}$ and $\mathrm{CH}$ as proxies for $\mathrm{H}_{2}$. To assess the reliability of this approach, a correlation analysis of $N(\mathrm{HF})$ and $N(\mathrm{CH})$ on the sightline to $\mathrm{W} 49 \mathrm{~N}$ was conducted (see Appendix E) and yields a correlation coefficient of 0.99 , with a false-alarm probability below $1 \%$.

Column densities of H I were obtained from JVLA data of the $\lambda 21 \mathrm{~cm}$ line (Winkel et al., in prep.).

\subsection{Distribution of $\mathrm{O}_{1}$}

Spectroscopically resolved observations of the ${ }^{3} \mathrm{P}_{1} \rightarrow{ }^{3} \mathrm{P}_{2}$ fine structure line of atomic oxygen, $\mathrm{O} I$, at $\lambda 63.2 \mu \mathrm{m}$ are rare. After the pioneering work of Melnick et al. (1979), who discovered the line in M17 and M42 with NASA's Lear Jet, Boreiko \& Betz (1996) resolved the line in M17 at $0.2 \mathrm{~km} \mathrm{~s}^{-1}$ channels spacing with laser heterodyne spectroscopy onboard the KAO. Such a high spectral resolution was out of reach for the ISO-LWS observations, with $\sim 35 \mathrm{~km} \mathrm{~s}^{-1}$. Nevertheless, Lis et al. (2001) successfully separated the O I absorption toward Sgr B2 into four components, three of which were found to be located in foreground clouds. By correcting the OI abundance in the atomic 

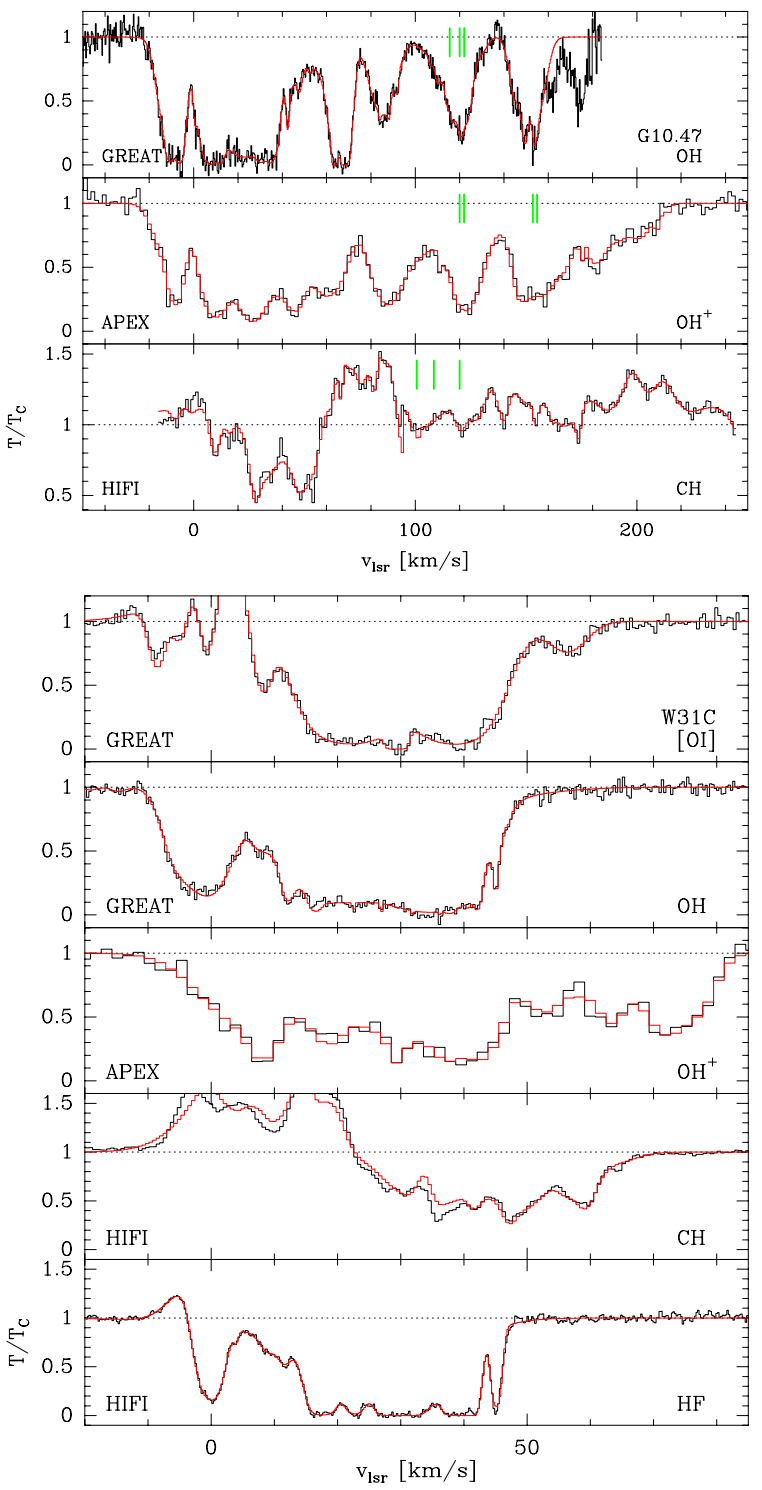

Fig. 1. $\mathrm{OI}^{3} \mathrm{P}_{1}-{ }^{3} \mathrm{P}_{2}$, $\mathrm{OH}{ }^{2} \Pi_{3 / 2} J=5 / 2 \leftarrow 3 / 2$ and $\mathrm{OH}^{+} N=1 \leftarrow 0$ spectra of sightlines in the first quadrant, along with $\mathrm{CH}^{2} \Pi_{3 / 2} J=$ $3 / 2 \leftarrow 1 / 2$ and HF $J=1 \leftarrow 0$ spectra. All five transitions are only available for W31C and W49N. Model fits are overlaid in red. For O I, $\mathrm{CH}$ and $\mathrm{HF}$ one emission line component was added where needed. For G10.47, the hyperfine splitting is indicated at the $120 \mathrm{~km} \mathrm{~s}^{-1}$ component (green bars). All spectra are normalized by the continuum level.

gas phase with existing $\mathrm{H}$ I data, the authors were able to establish an O I to CO abundance ratio of $\sim 9$, with a $50 \%$ error. The deduced $\mathrm{O}$ I abundance relative to the total hydrogen reservoir (i.e., atomic and molecular) amounts to $2.7 \times 10^{-4}$ in the dense molecular gas. Meanwhile, the spectroscopy of the ground-state lines of $\mathrm{HF}$ and of ${ }^{2} \Pi_{3 / 2} \mathrm{CH}$ has become accessible.

From the $\mathrm{O}$ I column densities shown in Fig. 3 and using HF as a proxy for $\mathrm{H}_{2}$, we obtain the correlation between $N(\mathrm{O} \mathrm{I})$ and $N(\mathrm{HI})+2 N\left(\mathrm{H}_{2}\right)$ shown in Fig. 6. Data points with $N / \sigma_{\mathrm{N}}<5$ in both quantities are discarded from the correlation analysis and the figure. This conservative cutoff implies that our analysis is not affected by saturated absorption. The false-alarm probabilities are below 5\%; here and in the following correlation analyses they are assumed to be given by Pearson's $p$-value. Ranging from 0 to 1 , it provides an estimate of the probability that the two quantities are uncorrelated (which is the null hypothesis,
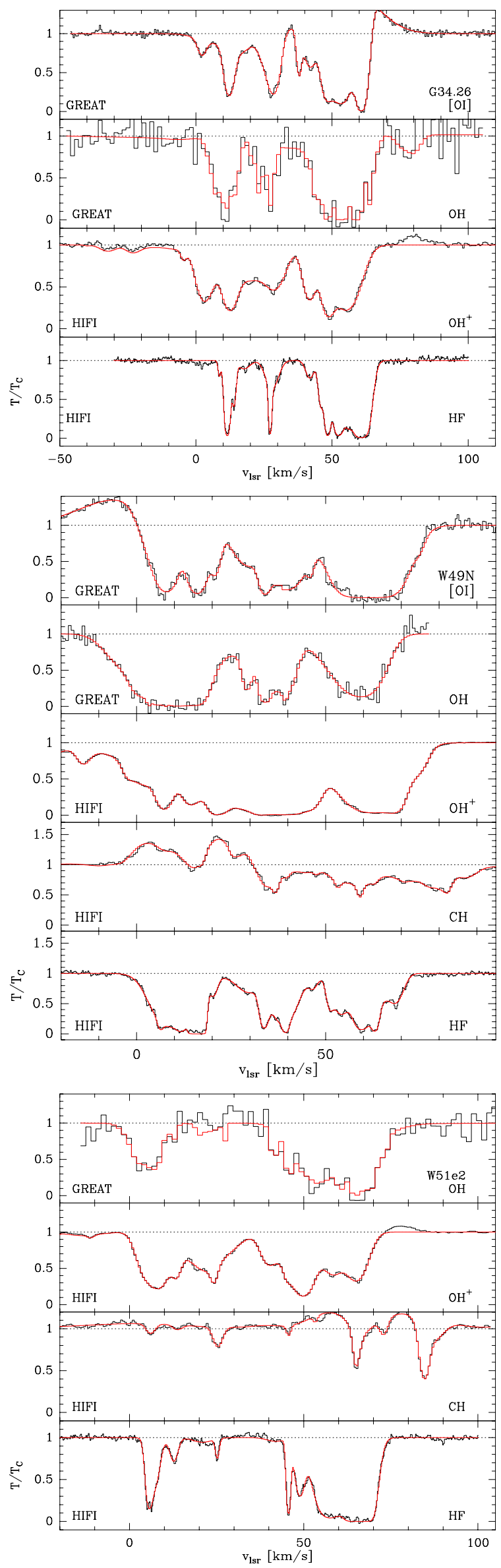

Fig. 1. continued. 
Table 4. Synoptic summary of results.

\begin{tabular}{|c|c|c|c|c|c|c|c|c|}
\hline Sightline & Spiral $\operatorname{arm}^{a}$ & $\begin{array}{l}v_{\min }, v_{\max } \\
{\left[\mathrm{km} \mathrm{s}^{-1}\right]}\end{array}$ & \multicolumn{2}{|c|}{$\left[10^{14} \mathrm{~cm}^{-2}\right]$} & $\begin{array}{c}N\left(\mathrm{H}_{2}\right)^{(b)} \\
{\left[10^{20} \mathrm{~cm}^{-2}\right]} \\
\end{array}$ & \multicolumn{2}{|c|}{$\times 10^{-7}$} & $\begin{array}{c}\mathrm{H}_{2} \\
\text { proxy }\end{array}$ \\
\hline \multirow[t]{3}{*}{ G10.47 } & & $(-20,0)$ & $5.5 \pm 1.8$ & $1.2 \pm 0.1$ & $10.7 \pm 0.2$ & $7.9 \pm 1.0$ & $4.33 \pm 0.11$ & $\mathrm{CH}$ \\
\hline & Sgr & $(0,+50)$ & $19 \pm 4$ & $4.8 \pm 0.3$ & $277 \pm 3$ & $0.83 \pm 0.17$ & $0.27 \pm 0.01$ & $\mathrm{CH}$ \\
\hline & Gal. bar & $(+80,+170)$ & $7.3 \pm 0.3$ & $4.6 \pm 0.1$ & $1167 \pm 8$ & $0.107 \pm 0.001$ & $0.07 \pm 0.01$ & $\mathrm{CH}$ \\
\hline \multirow{4}{*}{$\mathrm{G} 10.62^{d}$} & Sgr & $(+7,+15)$ & $0.95 \pm 0.01$ & $0.67 \pm 0.04$ & $8.21 \pm 0.09$ & $1.25 \pm 0.02$ & $1.12 \pm 0.09$ & $\mathrm{HF}$ \\
\hline & Sgr & $(+42,+55)$ & $1.52 \pm 0.05$ & $0.25 \pm 0.02$ & $11.24 \pm 0.26$ & $1.47 \pm 0.13$ & $0.14 \pm 0.01$ & $\mathrm{CH}$ \\
\hline & & & & & $12.08 \pm 0.34$ & $2.82 \pm 0.79$ & $0.28 \pm 0.02$ & $\mathrm{HF}$ \\
\hline & Scutum & $(+65,+80)$ & - & $0.22 \pm 0.01$ & & & & \\
\hline \multirow[t]{4}{*}{ G34.26 } & Sgr & $(0,+15)$ & $1.34 \pm 0.15$ & $0.43 \pm 0.02$ & $14.41 \pm 0.47$ & $5.1 \pm 6.4$ & $0.69 \pm 0.59$ & $\mathrm{HF}$ \\
\hline & Sgr & $(+15,+30)$ & $<0.001$ & $0.26 \pm 0.01$ & $9.39 \pm 0.23$ & $<0.005$ & $1.05 \pm 0.14$ & $\mathrm{HF}$ \\
\hline & & $(+38,+46)$ & $0.37 \pm 0.01$ & $0.20 \pm 0.01$ & $3.39 \pm 0.07$ & $0.59 \pm 0.02$ & $1.35 \pm 0.54$ & $\mathrm{HF}$ \\
\hline & & $(+62,+85)$ & $5 \pm 13$ & $0.023 \pm 0.001$ & $13.19 \pm 0.67$ & $49 \pm 69$ & $0.048 \pm 0.027$ & $\mathrm{HF}$ \\
\hline \multirow[t]{6}{*}{ W49N } & interarm & $(+20,+30)$ & $0.83 \pm 0.18$ & $0.93 \pm 0.01$ & $22.9 \pm 0.3$ & $0.33 \pm 0.03$ & $0.40 \pm 0.01$ & $\mathrm{CH}$ \\
\hline & & & & & $4.2 \pm 0.1$ & $2.22 \pm 0.20$ & $2.79 \pm 0.10$ & $\mathrm{HF}$ \\
\hline & $\operatorname{Sgr}^{e}$ & $(+30,+45)$ & $3.9 \pm 0.1$ & $2.17 \pm 0.01$ & $39.3 \pm 0.9$ & $0.93 \pm 0.03$ & $0.67 \pm 0.02$ & $\mathrm{CH}$ \\
\hline & & & & & $36.6 \pm 0.7$ & $1.06 \pm 0.02$ & $0.85 \pm 0.01$ & $\mathrm{HF}$ \\
\hline & $\operatorname{Sgr}^{f}$ & $(+45,+70)$ & $4.0 \pm 0.1$ & $1.8 \pm 0.1$ & $70.3 \pm 0.5$ & $0.55 \pm 0.01$ & $0.42 \pm 0.01$ & $\mathrm{CH}$ \\
\hline & & & & & $46.6 \pm 0.5$ & $1.54 \pm 0.09$ & $2.11 \pm 0.17$ & $\mathrm{HF}$ \\
\hline \multirow[t]{5}{*}{ W51e2 } & Sgr & $(0,+15)$ & $1.4 \pm 0.2$ & $0.43 \pm 0.03$ & $28.5 \pm 7.0$ & $0.49 \pm 0.11$ & $0.16 \pm 0.04$ & $\mathrm{CH}$ \\
\hline & & & & & $11.6 \pm 0.1$ & $4.41 \pm 4.47$ & $1.30 \pm 0.96$ & $\mathrm{HF}$ \\
\hline & & $(+15,+35)$ & $<0.1$ & $0.28 \pm 0.03$ & $1.32 \pm 0.07$ & $<1.0$ & $2.92 \pm 0.72$ & $\mathrm{HF}$ \\
\hline & & $(+40,+50)$ & $0.91 \pm 0.04$ & $0.37 \pm 0.01$ & $25.9 \pm 5.1$ & $0.28 \pm 0.06$ & $0.16 \pm 0.02$ & $\mathrm{CH}$ \\
\hline & & & & & $10.5 \pm 0.2$ & $1.17 \pm 0.15$ & $1.39 \pm 0.14$ & $\mathrm{HF}$ \\
\hline G327.29 & Carina & $(-25,+10)$ & $2.6 \pm 0.1$ & $0.58 \pm 0.01$ & $37.1 \pm 19.8$ & $1.86 \pm 0.77$ & $0.77 \pm 0.43$ & $\mathrm{CH}$ \\
\hline \multirow[t]{3}{*}{ G330.95 } & Crux & $(-70,-50)$ & $4.0 \pm 0.3$ & $0.81 \pm 0.02$ & $89 \pm 12$ & $0.39 \pm 0.04$ & $0.09 \pm 0.01$ & $\mathrm{CH}$ \\
\hline & Crux & $(-50,-25)$ & $11 \pm 1$ & $0.64 \pm 0.01$ & $84.4 \pm 7.6$ & $0.77 \pm 0.09$ & $0.16 \pm 0.01$ & $\mathrm{CH}$ \\
\hline & Carina & $(-25,+5)$ & $0.027 \pm 0.005$ & $0.08 \pm 0.01$ & $0.80 \pm 0.19$ & $0.37 \pm 0.11$ & $0.97 \pm 0.84$ & $\mathrm{CH}$ \\
\hline \multirow[t]{3}{*}{ G332.83 } & Norma & $(-74,-65)$ & $0.20 \pm 0.11$ & $0.007 \pm 0.001$ & & & & \\
\hline & Crux & $(-38,-30)$ & $0.55 \pm 0.06$ & $0.31 \pm 0.02$ & & & & \\
\hline & Crux & $(-30,-10)$ & $1.7 \pm 0.1$ & $0.55 \pm 0.01$ & & & & \\
\hline \multirow[t]{2}{*}{ G351.58 } & Crux, Norma & $(-65,-35)$ & $3.3 \pm 0.2$ & $1.0 \pm 0.1$ & & & & \\
\hline & Carina & $(-5,+5)$ & $0.42 \pm 0.12$ & $0.42 \pm 0.02$ & & & & \\
\hline
\end{tabular}

Notes. Column densities are integrated across the velocity intervals given in Col. 3. Abundances are mean values across the indicated velocity intervals. Data affected by saturated absorption are not considered. ${ }^{(a)}$ Spiral arms: Sgr/Car Sagittarius-Carina arm, Crux/Scu Crux-Scutum arm; (b) using $[\mathrm{CH}] /\left[\mathrm{H}_{2}\right]=3.5 \times 10^{-8}$ (Sheffer et al. 2008) and $[\mathrm{HF}] /\left[\mathrm{H}_{2}\right]=1.4 \times 10^{-8}$ (cf. Appendix E); ${ }^{(c)}$ velocity-interval averaged abundances with respect to $\mathrm{H}_{2}$; ${ }^{(d)}$ alias $\mathrm{W} 31 \mathrm{C}$ (the $\mathrm{CH}$ spectrum was not used for the $v_{\mathrm{lsr}}=(7,15) \mathrm{km} \mathrm{s}^{-1}$ interval due to blend with emission); ${ }^{(e)}$ near- and far-side crossing of Sagittarius arm; ${ }^{(f)}$ far-side crossing.

Table 5. Abundances of $\mathrm{OH}$ and of $\mathrm{OH}^{+}$with respect to $\mathrm{O}$.

\begin{tabular}{cccccc}
\hline \hline & $\begin{array}{c}T_{\mathrm{c}}{ }^{a} \\
{[\mathrm{~K}]}\end{array}$ & $\begin{array}{c}v_{\min }, v_{\max } \\
{\left[\mathrm{km} \mathrm{s}^{-1}\right]}\end{array}$ & $\begin{array}{c}N(\mathrm{OI}) \\
{\left[10^{18} \mathrm{~cm}^{-2}\right]}\end{array}$ & $f_{\mathrm{H}_{2}}^{\mathrm{N}}$ & $\frac{N(\mathrm{OH})^{b}}{N\left(\mathrm{OH}^{+}\right)}$ \\
\hline $\mathrm{G} 10.62$ & 2.6 & $(7,15)$ & $1.71 \pm 0.02$ & $0.210 \pm 0.004$ & $1.84 \pm 0.07$ \\
& & $(42,55)$ & $2.02 \pm 0.04$ & $0.144 \pm 0.005$ & $5.54 \pm 0.43$ \\
$\mathrm{G} 34.26$ & 3.0 & $(0,15)$ & $1.62 \pm 0.02$ & $0.133 \pm 0.004$ & $3.87 \pm 0.49$ \\
& & $(15,30)$ & $1.78 \pm 0.02$ & $0.079 \pm 0.002$ & $<0.004$ \\
W49N & 3.5 & $(38,46)$ & $1.19 \pm 0.01$ & $0.090 \pm 0.002$ & $1.69 \pm 0.03$ \\
& & $(30,30)$ & $1.33 \pm 0.02$ & $0.145 \pm 0.003$ & $0.82 \pm 0.06$ \\
& & $(45,60)$ & $5.45 \pm 0.11$ & $0.271 \pm 0.004$ & $1.68 \pm 0.02$ \\
& & & & & \\
& & & & & \\
\hline
\end{tabular}

Notes. Velocity intervals and spiral arm assignments as in Table 4 . With the following exceptions: G10.62 (alias W31C) - The $v_{\text {lsr }}=$ $(65,80) \mathrm{km} \mathrm{s}^{-1}$ interval from Table 4 is not reported because only $\mathrm{OH}^{+}$was found there. G34.26 - The $v_{\mathrm{lsr}}=(62,85) \mathrm{km} \mathrm{s}{ }^{-1}$ interval from Table 4 is not reported because the O Iline turns into emission there. W49N - The velocity interval for the far-side crossing of the Sagittarius spiral arm is only taken up to $v_{\text {lsr }}=60 \mathrm{~km} \mathrm{~s}^{-1}$ because the $\mathrm{O}$ I absorption beyond is saturated. ${ }^{(a)}$ Main-beam temperature of $4.744 \mathrm{THz}$ continuum (Rayleigh-Jeans scale). ${ }^{(b)}$ Error estimates do not account for systematic uncertainties and are derived from $\chi^{2}$ of fits by formal error propagation.

e.g., Cowley 1995). From a weighted regression analysis we derive oxygen abundances (relative to the total hydrogen reservoir, atomic and molecular) of $X(\mathrm{OI})=(3.5 \pm 0.1),(3.2 \pm 0.1)$ and $(3.1 \pm 0.1) \times 10^{-4}$ for $\mathrm{W} 31 \mathrm{C}, \mathrm{G} 34.26$, and $\mathrm{W} 49 \mathrm{~N}$, respectively. Velocities where the absorption in $\mathrm{HF}$ or $\mathrm{OI}$ is saturated are excluded, as are those corresponding to the hot cores, where the assumption of a complete ground-state population does not hold anymore. Remarkably, the abundances along these very different sightlines agree within $2 \sigma$. Likewise, Meyer et al. (1998) determined from UV spectroscopy of the $\lambda 1356 \AA$ line toward 

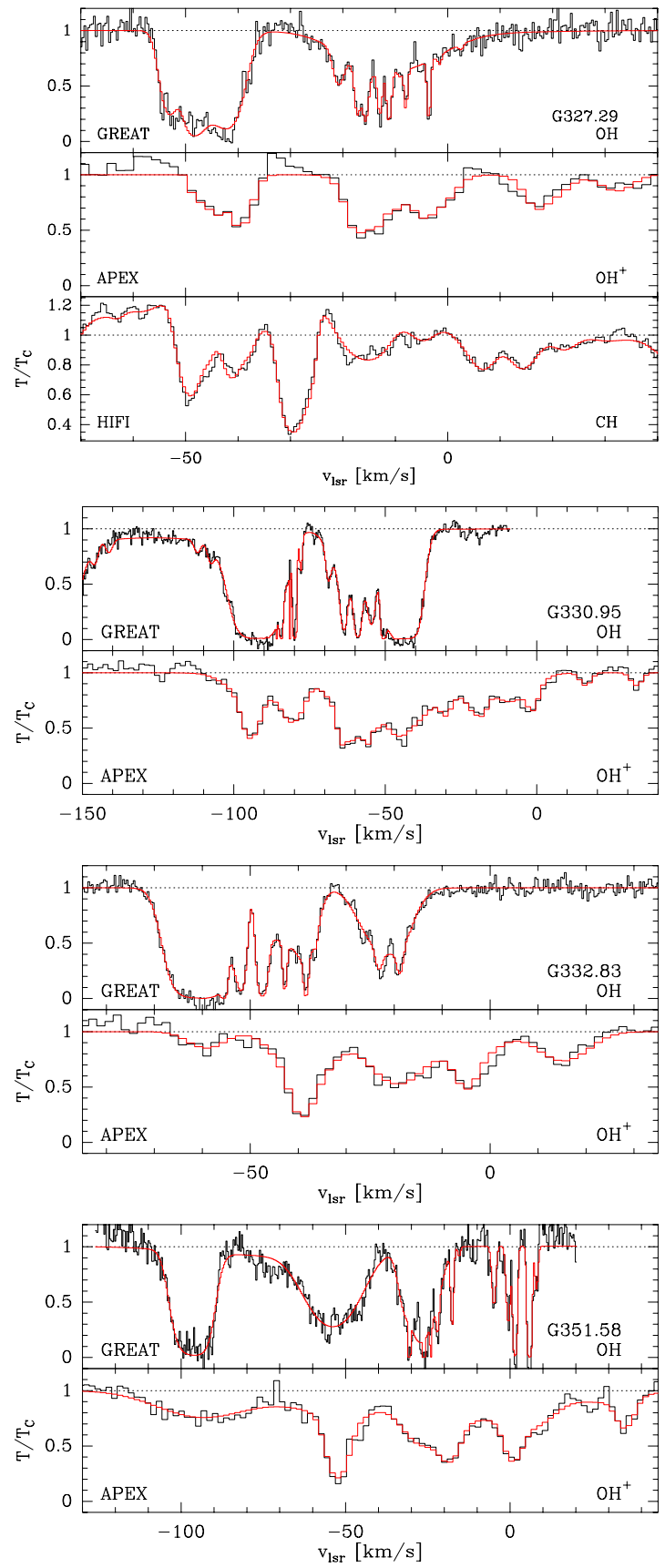

Fig. 2. Same as Fig. 1, but for sightlines in the fourth quadrant. In $\mathrm{G} 330.95$, the $\mathrm{OH}$ absorption at velocities below $-130 \mathrm{~km} \mathrm{~s}^{-1}$ is from the $\Lambda$ doublet in the other sideband and could be consistently fitted.

13 distinctly different sightlines tracing low-density diffuse gas $A_{\mathrm{V}} \lesssim 1.0 \mathrm{mag}$ an oxygen abundance of $(3.19 \pm 0.14) \times 10^{-4}$, in agreement with our result. Cartledge et al. (2004) later used the same technique on 36 sightlines and found oxygen abundances of $3.9 \times 10^{-4}$ on sightlines with lower mean density and $2.8 \times 10^{-4}$ on the denser ones. Jensen et al. (2005) determined a value of $4.2 \times 10^{-4}$ from their ten best-determined sightlines, with a $\sim 10 \%$ error. For the sake of comparison, we recall that the solar value was corrected downward over the years from $7 \times 10^{-4}$ (Pagel 1973, further references therein) to $4.9 \times 10^{-4}$ (Asplund et al. 2009).

All oxygen abundances are encompassed at the lower end by the value of Lis et al. (2001) for the denser, mainly molecular gas

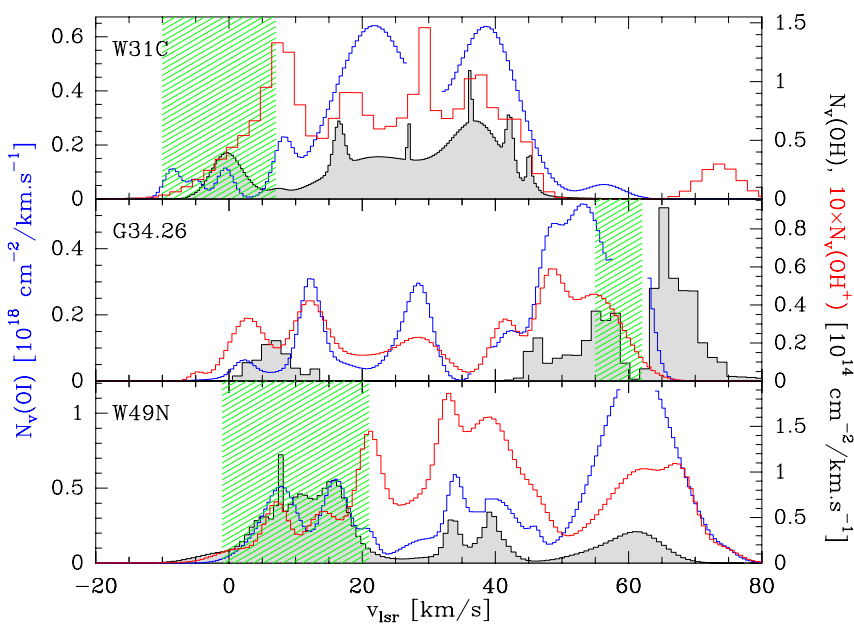

Fig. 3. Comparison of column densities of OI (blue), OH (black and gray filled) and $\mathrm{OH}^{+}$(red, scaled by a factor 10) for sightlines to continuum sources in the first quadrant. The velocity range spanned by $\mathrm{OH}$ and $\mathrm{CH}_{3} \mathrm{OH}$ masers in the hot cores is indicated by the greenhashed area. Velocity channels coinciding with the telluric or saturated O I absorption are masked.
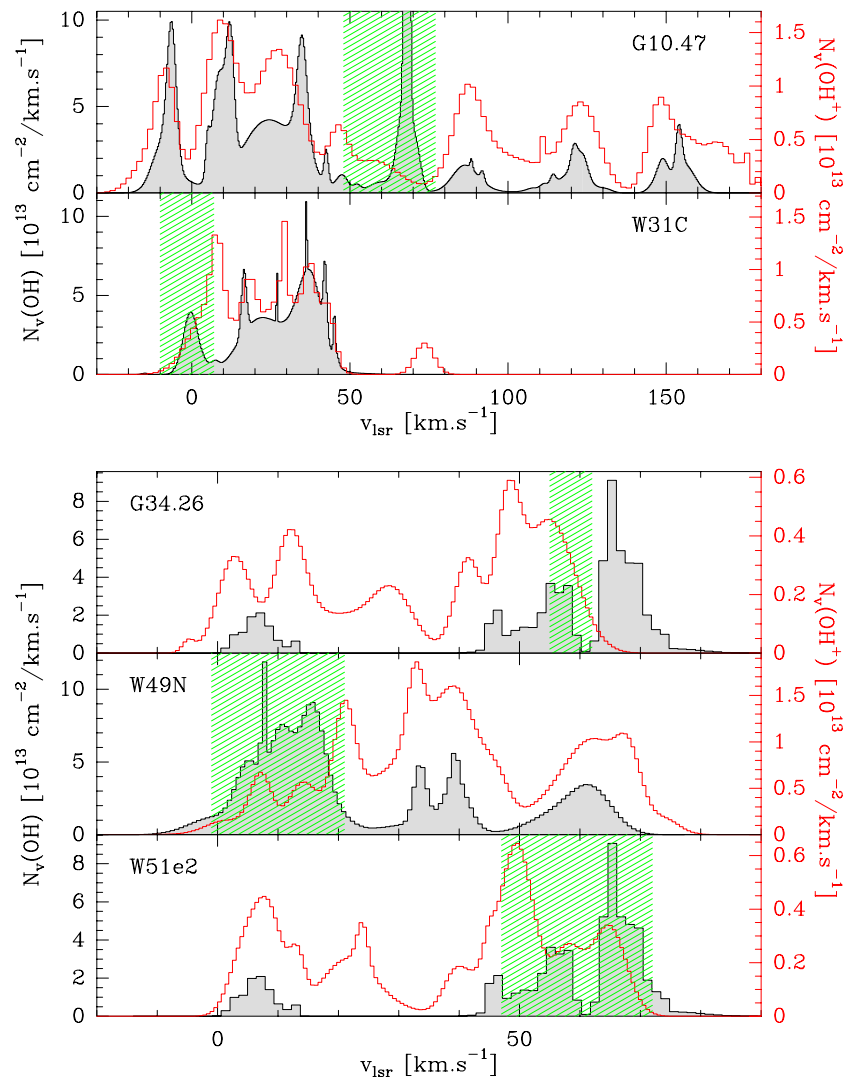

Fig. 4. Derived column density spectra of $\mathrm{OH}$ (gray filled histogram) and $\mathrm{OH}^{+}$(red lines) toward continuum sources in the first quadrant. Here and in the following figures $N_{\mathrm{V}}$ denotes the velocity-specific column density, i.e., the column density within a velocity interval is the area under the histograms. The green hashed area indicates the velocity range spanned by the $\mathrm{OH}$ and $\mathrm{CH}_{3} \mathrm{OH}$ masers in the hot cores where the derived column densities are uncertain due to saturated absorption and excitation of higher levels (for details see Table 2).

(but they are still compatible with our values within their error bars), and at the higher end by stellar abundances, for instance, $(5.75 \pm 0.4) \times 10^{-4}$ in the $\mathrm{B}$ stars of nearby OB associations 

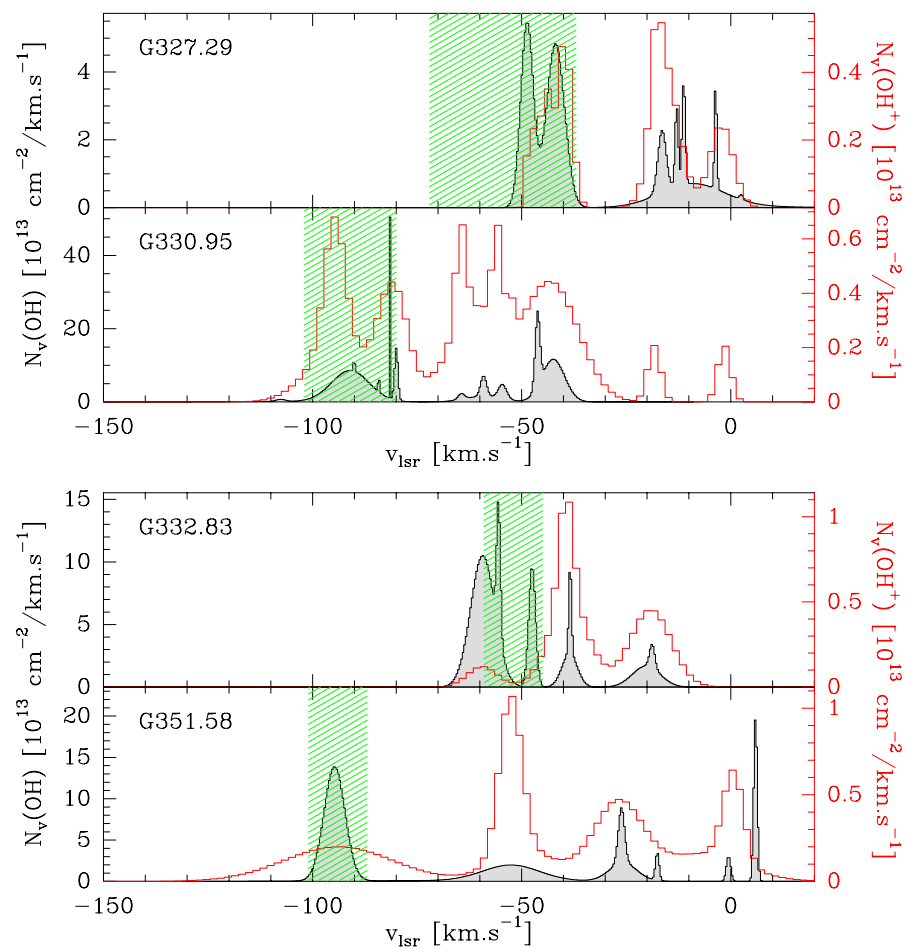

Fig. 5. As Fig. 4, but for sightlines to continuum sources in the fourth quadrant.

(Przybilla et al. 2008). The variation of abundances is partly due to inhomogeneities in the Galactic distribution of oxygen and partly due to the different analysis techniques. Observations from near-infrared to UV wavelengths need to apply corrections for interstellar extinction and non-LTE effects; neither correction is necessary in our approach using the $\lambda 63.2 \mu \mathrm{m}$ O I fine structure line. Abundances derived from ionized gas phases need to account for the resonant charge transfer reaction $\mathrm{O}^{+}\left(\mathrm{H}, \mathrm{H}^{+}\right) \mathrm{O}$ (e.g., Draine 2011), while our determination refers to the diffuse neutral gas phase, with an ionization fraction much smaller than unity (with $n_{\mathrm{e}} / n_{\mathrm{H}} \sim 10^{-4}$, mainly from the photoionization of carbon, e.g., van Dishoeck 1998). At the lower end of the determined oxygen abundances, that is, in clouds with a higher fraction of molecular hydrogen, the differences are seemingly easier to understand: At $A_{\mathrm{V}} \gtrsim 1.0 \mathrm{mag}$, the OIreservoir is used for the synthesis of oxygen-bearing species and for inclusion in the ice mantles of dust grains (while only some oxygen is fed back to the gas, e.g., through photodissociation of $\mathrm{OH}$ ). However, our picture of oxygen depletion is incomplete. Adopting as reference oxygen abundance the value of $(5.75 \pm 0.4) \times 10^{-4}$ of Przybilla et al. (2008), Whittet (2010) compared abundance measurements in various oxygen reservoirs to densities of up to $\sim 1000 \mathrm{~cm}^{-3}$ and found that an additional source of oxygen depletion is required at densities of $\gtrsim 10 \mathrm{~cm}^{-3}$. At the transition between diffuse and molecular clouds, where the opacity of the ISM precludes UV spectroscopy and where ice mantles start to form around dust grains, the author found that an oxygen abundance of $1.6 \times 10^{-4}$ cannot be attributed to either silicates or oxides. The carrier of this unidentified depleted oxygen (UDO) may be oxygen-bearing, organic matter. Jenkins (2009) concluded the same in a UV study of 17 elements on 243 sightlines. While this issue requires further investigation, we note that our oxygen abundance of $3 \times 10^{-4}$ precludes a strong increase of UDO at the densities sampled here (i.e., $n_{\mathrm{H}} \sim 100$ to $1000 \mathrm{~cm}^{-3}$ ), but does not rule out the presence of UDO either.
In summary, our abundance determination (on sightlines with molecular hydrogen fractions $f_{\mathrm{H}_{2}}^{\mathrm{n}} \lesssim 0.4$, with most data points below the value of 0.25 where half of the hydrogen atoms are bound in $\mathrm{H}_{2}$ ) is compatible with the somewhat larger oxygen abundance in gas that is predominantly atomic, in agreement with this picture. So far, only for W31C our data show an anti-correlation between the oxygen abundance and the molecular hydrogen fraction $f_{\mathrm{H}_{2}}^{\mathrm{N}}$ with a $p$-value $<0.05$ (Fig. 7; again, only data points with a relative error $<20 \%$ are retained). This significance may be taken as an indication of the depletion of O I with increasing molecular hydrogen density. However, the corresponding correlations on the sightlines to G34.26 and to W49N are not significant. As a caveat, we point out that these results depend on the adopted abundance of HF (which is the main surrogate for $\mathrm{H}_{2}$ ) and therefore on the underlying assumption that it is uniform along various sightlines. Therefore, these results are to be interpreted with caution. For G34.26 and W49N the correlations shown in Fig. 7 are insignificant, with $p$-values of 0.42 an 0.58 , respectively. This suggests that $\mathrm{O}$ I in the diffuse atomic and molecular gas is a tracer of both atomic and molecular hydrogen, except at the densities of translucent clouds and above. The formation of molecular oxygen as a source of depletion of $\mathrm{O}$ I is negligible here: While $\mathrm{O}_{2}$ has not been detected in the diffuse ISM, its abundance falls short of predictions by at least two orders of magnitude (Melnick \& Bergin 2005). Finally, we stress again that the $\mathrm{O}$ I abundances derived here are sightline-averaged quantities. The scatter in Figs. 6 and 7 might also be due to the galactocentric oxygen abundance gradient that is a natural consequence of oxygen production in short-lived, massive stars. In our sightlines the corresponding variation of the oxygen abundance may be of a factor of up to two: From stellar O II spectra, (Smartt \& Rolleston 1997) measure a gradient of $-0.07 \mathrm{dex} / \mathrm{kpc}$. From their non-LTE analysis of the near-IR O I triplet Korotin et al. (2014) obtained a similar gradient of $-0.058 \mathrm{dex} / \mathrm{kpc}$, and found

$\log [\mathrm{O} / \mathrm{H}]=-2.72-0.058 R_{\mathrm{G}}[\mathrm{kpc}]$.

For the solar $R_{\mathrm{G}}=8.34 \mathrm{kpc}$ (Reid et al. 2014), the local oxygen abundance amounts to $X(\mathrm{OI})=6.3 \times 10^{-4}$. The smallest galactocentric radius among our O I sightlines is that of $\mathrm{W} 31 \mathrm{C}$, $R_{\mathrm{G}}=3.6 \mathrm{kpc}$, where $X(\mathrm{OI})=1.2 \times 10^{-3}$ is expected. An attempt to determine the Galactic O I gradient from our groundstate data remained inconclusive because of the limited number of sightlines and the frequent saturated absorption, but should be possible with a dedicated, extended study.

\subsection{Chemistry of $\mathrm{OH}$ and $\mathrm{OH}^{+}$}

The median value of the column density of $\mathrm{OH}$ in the first quadrant is $3.9 \times 10^{14} \mathrm{~cm}^{-3}$ and for $\mathrm{OH}^{+}$it is $0.68 \times 10^{14} \mathrm{~cm}^{-3}$. In the fourth quadrant, the median column densities amount to $1.7 \times 10^{14} \mathrm{~cm}^{-3}$ and $0.55 \times 10^{14} \mathrm{~cm}^{-3}$, respectively. Column densities in excess of $10^{15} \mathrm{~cm}^{-2}$ (for $\mathrm{OH}$ ) and above $\sim 10^{14} \mathrm{~cm}^{-2}$ (for $\left.\mathrm{OH}^{+}\right)$are rather the exception. The median $N(\mathrm{OH}) / N\left(\mathrm{OH}^{+}\right)$ratio over all sightlines and velocity components is 3.3 . While the formation of $\mathrm{OH}^{+}$results from cosmic-ray induced reactions involving atomic and molecular hydrogen and atomic oxygen, the bottleneck expected for the formation of $\mathrm{OH}$ via ion-neutral chemistry is the availability of $\mathrm{H}_{2}$. The reaction of $\mathrm{OH}^{+}$with $\mathrm{H}_{2}$ yields $\mathrm{H}_{3} \mathrm{O}^{+}$, via the two hydrogen abstraction reactions $\mathrm{OH}^{+}\left(\mathrm{H}_{2}, \mathrm{H}\right) \mathrm{H}_{2} \mathrm{O}^{+}\left(\mathrm{H}_{2}, \mathrm{H}\right) \mathrm{H}_{3} \mathrm{O}^{+}$(see Appendix C). Then the dissociative recombination of $\mathrm{H}_{3} \mathrm{O}^{+}$yields $\mathrm{OH}$ and $\mathrm{H}_{2} \mathrm{O}$, with a branching ratio of $\sim 74 \%$ to $83 \%$ in favor of $\mathrm{OH}$ (determined 

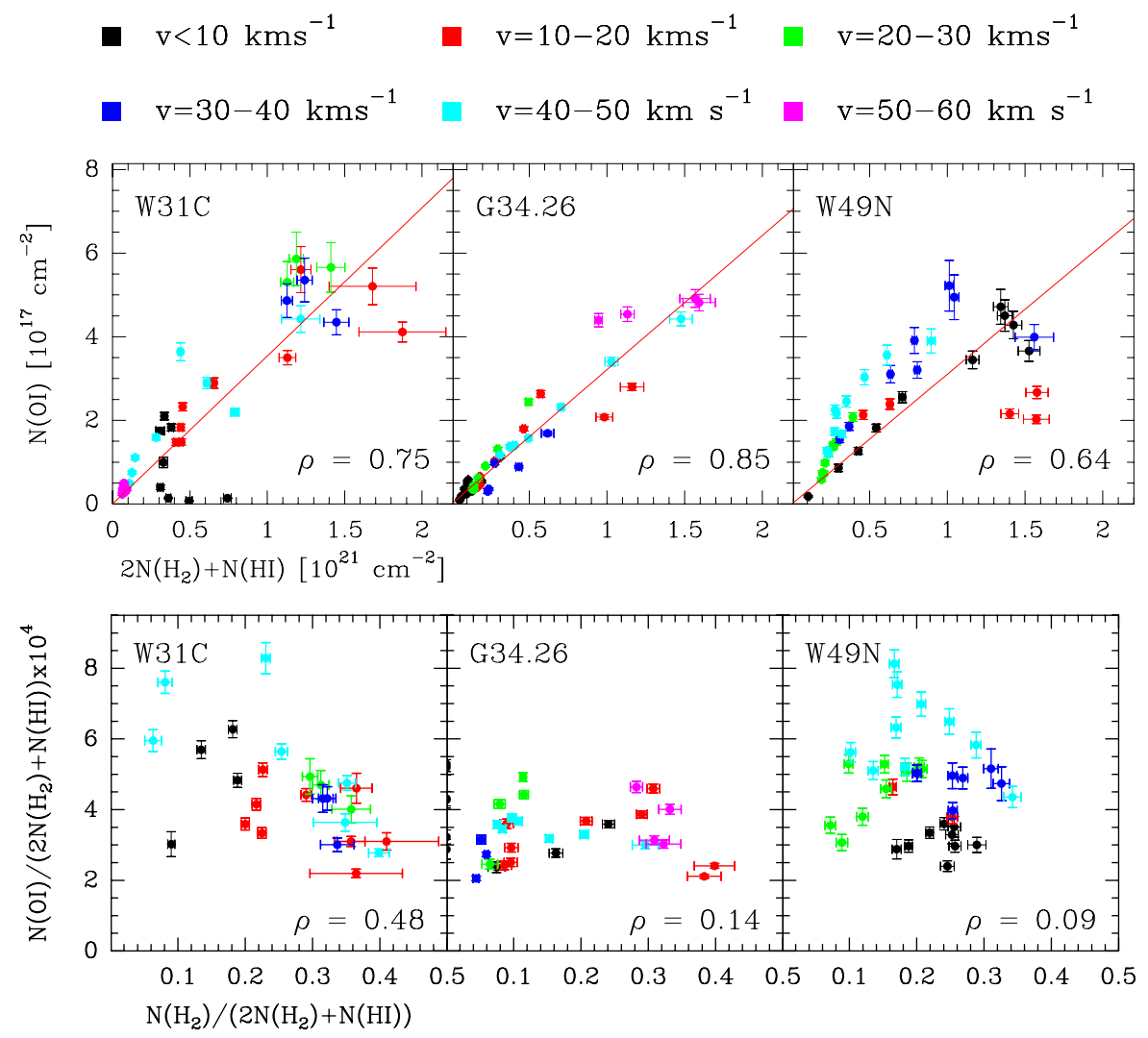

Fig. 6. Correlation between the O I column density and the total (atomic and molecular) hydrogen column density derived from $\mathrm{HF}$ and $\mathrm{H}$ I for sightlines toward W31C, G34.26, and W49N and in $1 \mathrm{~km} \mathrm{~s}^{-1}$ wide velocity bins. Colors indicate the velocity intervals defined in the legend. Only data points with $N / \sigma_{\mathrm{N}}>5$ both in OIand $2 N\left(\mathrm{H}_{2}\right)+N(\mathrm{H} \mathrm{I})$ are shown. The regression lines are shown in red. The corresponding correlation coefficients $\rho$ are given in the lower right corners. For details see text.

Fig. 7. Atomic oxygen abundance versus molecular hydrogen fraction $f_{\mathrm{H}_{2}}^{\mathrm{N}}$, for sightlines toward W31C, G34.26, and W49N and in $1 \mathrm{~km} \mathrm{~s}^{-1}$ wide velocity bins. Correlation coefficients are given in the lower right corners. The correlation is significant only for W31C. Colors indicate the velocity intervals defined in the legend of Fig. 6. Data points affected by saturated absorption are discarded. by ion storage ring experiments, Jensen et al. 2000; Neau et al. 2000), while less than $\sim 1 \%$ forms O I. In the following, we attempt to confirm these predictions, that is, the bottleneck reaction $\mathrm{OH}^{+}\left(\mathrm{H}_{2}, \mathrm{H}\right) \mathrm{H}_{2} \mathrm{O}^{+}$, and the $N(\mathrm{OH}) / N\left(\mathrm{H}_{2} \mathrm{O}\right)$ ratio. As for the former, a strong anticorrespondence between the column densities of $\mathrm{OH}$ and $\mathrm{OH}^{+}$might naively be expected, where the availability of $\mathrm{H}_{2}$ tips the scales in favor of $\mathrm{OH}$, while $\mathrm{OH}^{+}$traces predominantly atomic gas (Hollenbach et al. 2012, further references therein). But even if a clear anticorrelation between $\mathrm{OH}$ and $\mathrm{OH}^{+}$existed, it would be impossible to observe it. On a given sightline several clouds with high and low molecular hydrogen fractions $f_{\mathrm{H}_{2}}^{\mathrm{N}}=N\left(\mathrm{H}_{2}\right) /\left(2 N\left(\mathrm{H}_{2}\right)+N(\mathrm{H} \mathrm{I})\right)$ line up. Even across a single diffuse cloud, the $N(\mathrm{OH}) / N\left(\mathrm{OH}^{+}\right)$ratio is expected to vary substantially, depending on the degree of selfshielding of $\mathrm{H}_{2}$ against the interstellar UV radiation field. To quantify the anticorrelation, we normalized the velocity-specific $\mathrm{OH}$ column density with the total $\mathrm{OH}$ and $\mathrm{OH}^{+}$reservoir and obtained an abundance ratio $r=N_{\mathrm{v}}(\mathrm{OH}) /\left(N_{\mathrm{v}}(\mathrm{OH})+N_{\mathrm{v}}\left(\mathrm{OH}^{+}\right)\right.$ varying from zero (only $\mathrm{OH}^{+}$, no $\mathrm{OH}$ ) to one, where all the $\mathrm{OH}^{+}$abundance is exhausted owing to the formation of $\mathrm{OH}$ and (see below) water. (The normalization chosen here avoids the divergence of the distribution if $\mathrm{OH}$ has no spectral counterpart in $\mathrm{OH}^{+}$.) The resulting distribution (Fig. 12) indeed shows that these extremes are present in the data, although the second case is by an order of magnitude more frequent. We suggest two explanations for this. One reason is that if $f_{\mathrm{H}_{2}}^{\mathrm{N}}$ is too small, $\mathrm{OH}^{+}$can be efficiently destroyed by the dissociative recombination with free electrons (Appendix $\mathrm{C}$ ), while the formation of $\mathrm{OH}^{+}$by the reaction chain $\mathrm{H}^{+}(\mathrm{O}, \mathrm{H}) \mathrm{O}^{+}\left(\mathrm{H}_{2}, \mathrm{H}\right) \mathrm{OH}^{+}$and the secondary, less important path $\mathrm{H}_{2}{ }^{+}\left(\mathrm{H}_{2}, \mathrm{H}\right) \mathrm{H}_{3}{ }^{+}\left(\mathrm{O}, \mathrm{H}_{2}\right) \mathrm{OH}^{+}$become less efficient (see Appendix D) because less $\mathrm{H}_{2}$ is available. Another reason is that the fraction $f_{\mathrm{H}_{2}}^{\mathrm{N}}$ is larger in denser gas (cf. Table 3) where column densities are higher and absorption features easier to observe.
The anticorrelation between $N(\mathrm{OH})$ and $N\left(\mathrm{OH}^{+}\right)$is expected to increase with the fractional abundance of molecular hydrogen, $f_{\mathrm{H}_{2}}^{\mathrm{N}}$. Again using $\mathrm{HF}$ as surrogate for $\mathrm{H}_{2}$ with $X(\mathrm{HF})=$ $1.4 \times 10^{-8}$, for $\mathrm{W} 31 \mathrm{C}$ and $\mathrm{W} 49 \mathrm{~N}$ we indeed find a correlation between the $N(\mathrm{OH}) / N\left(\mathrm{OH}^{+}\right)$ratio (Fig. 12 shows that divergence of this ratio is excluded), with coefficients $\rho=0.42,0.02$ and 0.43 toward $\mathrm{W} 31 \mathrm{C}, \mathrm{G} 34.26$, and $\mathrm{W} 49 \mathrm{~N}$, respectively, and falsealarm probabilities of $6 \%, 94 \%$, and $3 \%$ (Fig. 8, again, only data points with relative error $<20 \%$ are retained). We note that qualitatively similar but less significant correlations can be deduced using $\mathrm{O} I$ instead of $\mathrm{HI}$ and $\mathrm{H}_{2}$, in agreement with the results shown in Sect. 4.1. The lack of a significant correlation toward G34.26 is most likely explained by the few data points available on this relatively short line of sight (1.56 kpc, see Appendix A). As for the sightline to $\mathrm{W} 31 \mathrm{C}$, the significant correlation has to be interpreted with care: The background source is located in the $3 \mathrm{kpc}$ arm where the density of Galactic free electrons is an order of magnitude above its value in the solar neighborhood (Gómez et al. 2001). In such an environment the $n\left(\mathrm{H}_{2}\right) / n\left(\mathrm{e}^{-}\right)$ratio falls short of $\sim 100$ (cf. Table C.1), so that neither $\mathrm{H}_{2} \mathrm{O}^{+}$nor the subsequent products can be formed owing to the dissociative recombination of $\mathrm{OH}^{+}$. Furthermore, the complex gas kinematics in the $3 \mathrm{kpc}$ arm (Sanna et al. 2014, further references therein) and the resulting confusion due to the sightline crowding mentioned above add to the complexity, and drawing more quantitative conclusions proves to be difficult. However, given that the correlation toward $\mathrm{W} 49 \mathrm{~N}$ is also significant, it seems fair to say that our results confirm the importance of the reaction $\mathrm{OH}^{+}\left(\mathrm{H}_{2}, \mathrm{H}\right) \mathrm{H}_{2} \mathrm{O}^{+}$and conclusions that $\mathrm{OH}^{+}$is rather associated with diffuse, atomic gas (Gerin et al. 2010b; Neufeld et al. 2010), while the $\mathrm{OH}$ seen in unsaturated absorption is located in diffuse molecular and translucent clouds. The underabundance of $\mathrm{OH}^{+}$ in the latter with respect to $\mathrm{OH}$ was also observed in UV spectroscopy (Krełowski et al. 2010). 


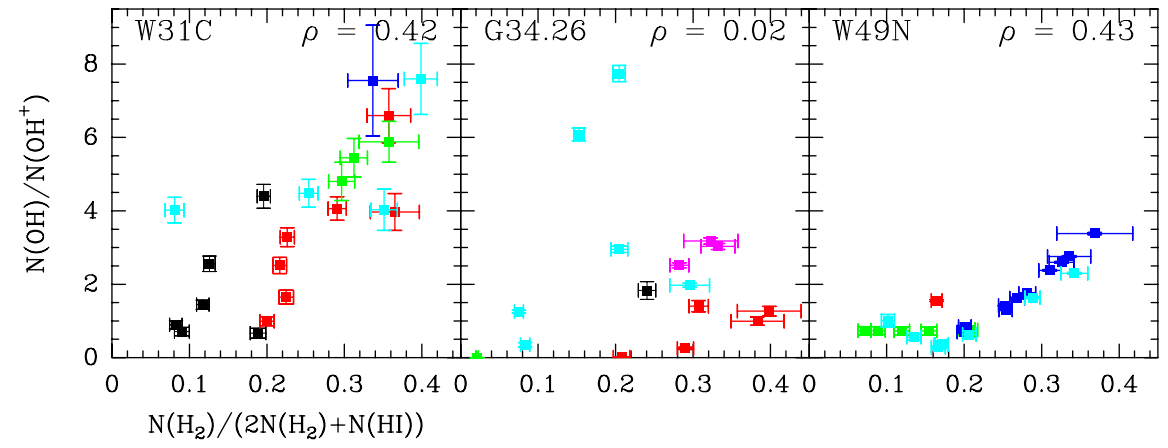

Fig. 8. Correlation between the $N(\mathrm{OH}) / N\left(\mathrm{OH}^{+}\right)$ ratio and the molecular hydrogen fraction $f_{\mathrm{H}_{2}}^{\mathrm{N}}=$ $N\left(\mathrm{H}_{2}\right) /\left(N(\mathrm{HI})+2 N\left(\mathrm{H}_{2}\right)\right)$ for $\mathrm{W} 31 \mathrm{C}, \mathrm{G} 34.26$, and $\mathrm{W} 49 \mathrm{~N}$. Colors indicate the velocity intervals defined in the legend of Fig. 6. Data points affected by saturated absorption are discarded. Correlation coefficients $\rho$ are given in the upper right corners. For details see text.
We derived $\mathrm{OH}$ abundances $X(\mathrm{OH}) \sim 10^{-8}$ up to $\sim 10^{-6}$, and $\mathrm{OH}^{+}$abundances from $\sim 10^{-8}$ to $\sim 10^{-7}$. The smallest and largest $\mathrm{OH}$ abundances were encountered on the sightline to G10.47, in the $135 \mathrm{~km} \mathrm{~s}^{-1}$ arm and the Sagittarius arm, respectively. Both values are somewhat uncertain because only $\mathrm{CH}$ is available as $\mathrm{H}_{2}$ tracer and, for the $135 \mathrm{~km} \mathrm{~s}^{-1}$ arm, the same caveat holds as for the environment of W31C. The $\mathrm{OH}$ abundances for the remaining sightlines agree reasonably well (i.e., within error bars) with the values predicted by Albertsson et al. (2014), who modeled the chemistry in diffuse clouds, including the time-dependence of the ortho-to-para ratio of $\mathrm{H}_{2}, \mathrm{H}_{2}{ }^{+}$and $\mathrm{H}_{3}{ }^{+}$, gas-grain interactions and grain surface reactions. They obtained $X(\mathrm{OH})=(0.3-1.6) \times 10^{-7}$, based on the chemical model underlying the Meudon PDR code (Le Petit et al. 2006), which includes reactions on the surface of dust grains. The models of Albertsson et al. (2014) do not include the endothermic reactions of warm chemistry, triggered by turbulent dissipation regions (TDRs) or slow shocks, while the precision of our OH abundance determinations is not good enough to exclude them. An additional piece of information is the production of water by the following reaction chain: $\mathrm{O}\left(\mathrm{H}_{2}, \mathrm{H}\right) \mathrm{OH}\left(\mathrm{H}_{2}, \mathrm{H}\right) \mathrm{H}_{2} \mathrm{O}$ with energy barriers of $2980 \mathrm{~K}$ and $1490 \mathrm{~K}$. This warm chemistry was investigated by Godard et al. (2012). According to the authors, the models with lower rates of turbulent strain and thus dominated by ion-neutral drift yield the best description of the observed abundance pattern. Thanks to the aforementioned highly endothermic path to the production of $\mathrm{OH}$ and $\mathrm{H}_{2} \mathrm{O}$, the abundance ratio of $X\left(\mathrm{H}_{2} \mathrm{O}\right) / X(\mathrm{OH})$ is sensitive to the dissipation of turbulence. Godard et al. (2012) obtained a typical ratio of 0.16 for a UV shielding of $A_{\mathrm{V}}=0.4 \mathrm{mag}$ and $n_{\mathrm{H}}=100 \mathrm{~cm}^{-3}$. Comparing our $\mathrm{OH}$ column densities with those determined for para-water by Sonnentrucker et al. (2010), we obtain for their velocity intervals the correlation shown in Fig. 13. The ortho/para ratio in translucent clouds was determined by Flagey et al. (2013) to be $3: 1$, its equilibrium value in the high-temperature limit. The resulting abundance ratio is $X\left(\mathrm{H}_{2} \mathrm{O}\right) / X(\mathrm{OH})=0.28$, which is reasonably similar to the value for chemistry driven by turbulence.

\subsubsection{Branching ratio of the dissociative recombination of $\mathrm{H}_{3} \mathrm{O}^{+}$}

We finally attempt to determine the branching ratio of the dissociative recombination of $\mathrm{H}_{3} \mathrm{O}^{+}$, forming $\mathrm{OH}$ and $\mathrm{H}_{2} \mathrm{O}$. If the chemistry is dominated by cold ion-neutral reactions, the ratio between $X\left(\mathrm{H}_{2} \mathrm{O}\right) / X\left(\mathrm{H}_{3} \mathrm{O}^{+}\right)$and $X(\mathrm{OH}) / X\left(\mathrm{H}_{3} \mathrm{O}^{+}\right)$should reflect the measured branching ratios of 74 to $83 \%$ (Jensen et al. 2000; Neau et al. 2000). We adopt the following phenomenological description of the underlying chemistry, with the rate equations

$$
\begin{aligned}
& \frac{\mathrm{d} n_{\mathrm{OH}}}{\mathrm{d} t}=\Gamma_{\mathrm{OH}}+\beta \gamma_{\mathrm{DR}} n_{\mathrm{H}_{3} \mathrm{O}^{+}}+\gamma_{\mathrm{PD}} n_{\mathrm{H}_{2} \mathrm{O}}-\Lambda_{\mathrm{OH}} n_{\mathrm{OH}}, \\
& \frac{\mathrm{d} n_{\mathrm{H}_{2} \mathrm{O}}}{\mathrm{d} t}=\Gamma_{\mathrm{H}_{2} \mathrm{O}}+(1-\beta) \gamma_{\mathrm{DR}} n_{\mathrm{H}_{3} \mathrm{O}^{+}}-\left(\Lambda_{\mathrm{H}_{2} \mathrm{O}}+\gamma_{\mathrm{PD}}\right) n_{\mathrm{H}_{2} \mathrm{O}},
\end{aligned}
$$

where $\gamma_{\mathrm{DR}}$ and $\gamma_{\mathrm{PD}}$ are the rates for the dissociative recombination of $\mathrm{H}_{3} \mathrm{O}^{+}$and, respectively, the photodissociation of $\mathrm{H}_{2} \mathrm{O}$. Gains and losses in the populations of $\mathrm{OH}$ and $\mathrm{H}_{2} \mathrm{O}$ that are not due to these two processes are denoted $\Gamma$ and $\Lambda$, respectively. The branching ratio for the dissociative recombination of $\mathrm{H}_{3} \mathrm{O}^{+}$ is denoted $\beta . \mathrm{H}_{3} \mathrm{O}^{+}$is difficult to observe, and only one measurement is available (in the $(55,75) \mathrm{km} \mathrm{s}^{-1}$ interval toward W51e2 Indriolo et al. 2015). We therefore used $\mathrm{H}_{2} \mathrm{O}^{+}$instead, with the working hypothesis that its abundance is proportional to that of $\mathrm{H}_{3} \mathrm{O}^{+}$. For equilibrium chemistry, this assumption is possibly justified. From our data and those published by Sonnentrucker et al. $(2010,2015)$ for $\mathrm{H}_{2} \mathrm{O}$ and by Indriolo et al. (2015) for $\mathrm{H}_{2} \mathrm{O}^{+}$we derive significant linear correlations between the column densities of $\mathrm{OH}$ and $\mathrm{H}_{2} \mathrm{O}^{+}$, and between those of $\mathrm{H}_{2} \mathrm{O}$ and $\mathrm{H}_{2} \mathrm{O}^{+}$, with correlation coefficients of, respectively, 0.98 (falsealarm probability $<1 \%$ ) and 0.87 (false-alarm probability $5 \%$ ). Using Eq. (6), the ratio of the slopes $b_{\mathrm{OH}}$ and $b_{\mathrm{H}_{2} \mathrm{O}}$ derived from the linear regression analysis is

$\frac{b_{\mathrm{OH}}}{b_{\mathrm{H}_{2} \mathrm{O}}}=\frac{\gamma_{\mathrm{PD}}+\beta \Lambda_{\mathrm{H}_{2} \mathrm{O}}}{(1-\beta) \Lambda_{\mathrm{OH}}}$.

Identifying photoionization and photodissociation as main loss channels for the abundances of $\mathrm{OH}$ and $\mathrm{H}_{2} \mathrm{O}$ (cf. Table C.1), we find

$$
\begin{aligned}
\gamma_{\mathrm{PD}}\left[\mathrm{s}^{-1}\right]= & 7.5 \times 10^{-10} \exp \left(-1.70 A_{\mathrm{v}}\right) \\
\Lambda_{\mathrm{H}_{2} \mathrm{O}}\left[\mathrm{s}^{-1}\right]= & 3.1 \times 10^{-11} \exp \left(-3.90 A_{\mathrm{V}}\right)+4.8 \times 10^{-11} \exp \left(-2.20 A_{\mathrm{V}}\right), \\
\Lambda_{\mathrm{OH}}\left[\mathrm{s}^{-1}\right]= & 2.2 \times 10^{-11} \exp \left(-4.05 A_{\mathrm{V}}\right)+3.9 \times 10^{-10} \exp \left(-1.70 A_{\mathrm{V}}\right) \\
& +2.9 \times 10^{-9}(T / 300 \mathrm{~K})^{-0.33} n_{\mathrm{C}^{+}}\left[\mathrm{cm}^{3}\right]
\end{aligned}
$$

The first term on the right-hand side of the loss rate for $\mathrm{H}_{2} \mathrm{O}$ is due to its photoionization, the second one due to the photodissociation of $\mathrm{H}_{2} \mathrm{O}$ to $\mathrm{OI}$. Likewise, the first two terms on the right-hand side of the loss rate of $\mathrm{OH}$ are due to photoionization and to photodissociation, respectively, while the last contribution to the loss channels of $\mathrm{OH}$ comes from the ion-neutral reaction $\mathrm{C}^{+}(\mathrm{OH}, \mathrm{H}) \mathrm{CO}^{+}$, for which we assumed a $\mathrm{C}^{+}$density of $n_{\mathrm{C}^{+}} \sim 0.01 \mathrm{~cm}^{-3}$, in agreement with the models shown in Appendix D and Table 3. The deduced branching ratios are shown in Fig. 11 for visual extinctions and gas temperatures in the range of our models. The underlying column densities are shown in Table 6 . Up to $A_{\mathrm{V}}=1.0 \mathrm{mag}$ the branching ratio depends only weakly on temperature. For the regime of our models we obtain a branching ratio of $0.84-0.91$. This is compatible with the value of 0.83 determined by Neau et al. (2000). We note that the $\mathrm{p}-\mathrm{H}_{2} \mathrm{O}$ column densities of Sonnentrucker et al. (2010) were again corrected by the ortho/para ratio of 3:1, and the same ortho/para ratio for $\mathrm{H}_{2} \mathrm{O}$ and $\mathrm{H}_{2} \mathrm{O}^{+}$was assumed. 


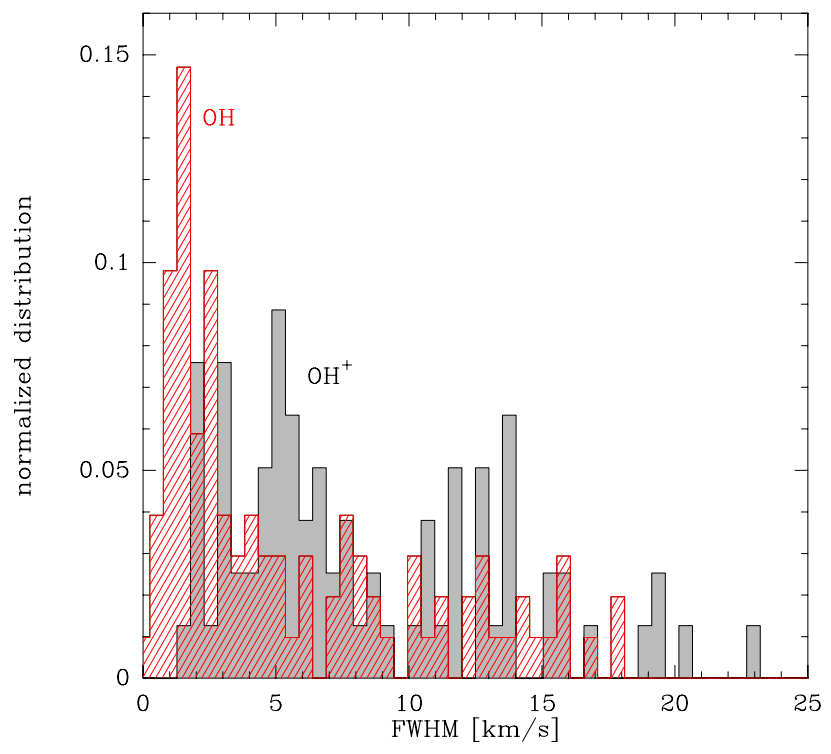

Fig. 9. Normalized distribution of the velocity widths (FWHM) of Gaussian velocity components (red: $\mathrm{OH}$, gray: $\mathrm{OH}^{+}$).

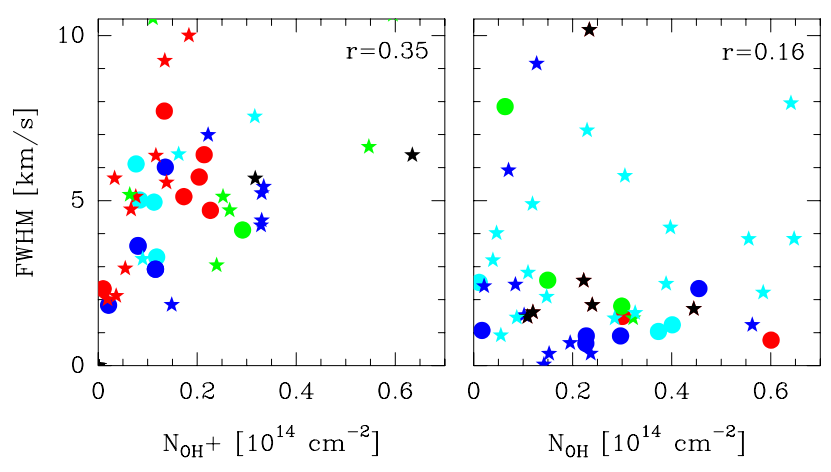

Fig. 10. Comparison of line widths (ordinate) with column densities (abscissa) of the fitted Gaussian velocity components. The circles are for sightlines in the first quadrant, the stars for sightlines in the fourth quadrant. Each color represents a sightline toward a different target. Left: $\mathrm{OH}^{+}$, right: $\mathrm{OH}$.

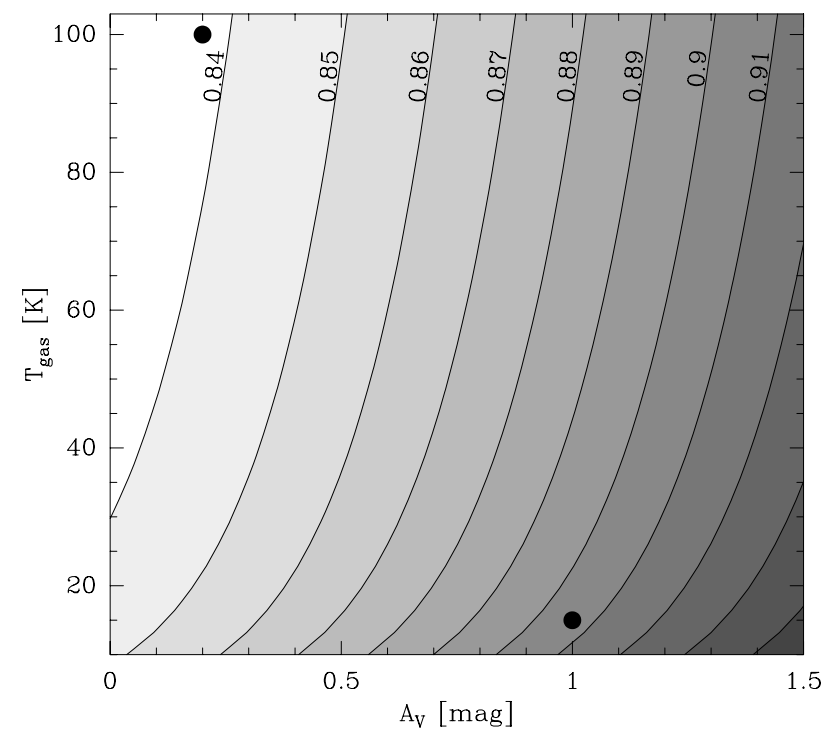

Fig. 11. Branching ratio (contour label) for the dissociative recombination of $\mathrm{H}_{3} \mathrm{O}^{+}$to $\mathrm{OH}$ and $\mathrm{H}_{2} \mathrm{O}$ as a function of visual extinction, $A_{\mathrm{v}}$, and gas temperature for an ortho-to-para ratio of $\mathrm{H}_{2} \mathrm{O}$ and of $\mathrm{H}_{2} \mathrm{O}^{+}$of 3:1. Column densities of $\mathrm{H}_{2} \mathrm{O}$ are from Sonnentrucker et al. (2010, 2015). The two black dots are at the parameters for models 1 and 2. For details see text.
Table 6. Column densities of $\mathrm{p}-\mathrm{H}_{2} \mathrm{O}, \mathrm{H}_{2} \mathrm{O}^{+}$and $\mathrm{OH}$ used in the determination of the branching ratio.

\begin{tabular}{cccc}
\hline \hline $\begin{array}{c}v_{\min }, v_{\max } \\
{\left[\mathrm{km} \mathrm{s}^{-1}\right]}\end{array}$ & $N(\mathrm{OH})$ & $\begin{array}{c}N\left(p-\mathrm{H}_{2} \mathrm{O}\right)^{a} \\
{\left[10^{13} \mathrm{~cm}^{-2}\right]}\end{array}$ & $N\left(p-\mathrm{H}_{2} \mathrm{O}^{+}\right)^{b}$ \\
\hline \multicolumn{3}{c}{$\mathrm{W} 49 \mathrm{~N}$} \\
\hline $30-50$ & $4.0 \pm 0.1$ & $2.2 \pm 0.8$ & $5.3 \pm 0.4$ \\
$50-78$ & $3.9 \pm 0.1$ & $3.4 \pm 0.9$ & $2.4 \pm 0.2$ \\
$67-71$ & $0.17 \pm 0.04$ & $0.15 \pm 0.03$ & $0.8 \pm 0.1$ \\
\hline \multicolumn{3}{c}{$\mathrm{W} 51$} \\
\hline $0-10$ & $11.4 \pm 0.9$ & $0.62 \pm 0.05$ & $0.6 \pm 0.1$ \\
$10-20$ & $2 \pm 1$ & $0.04 \pm 0.01$ & $0.26 \pm 0.03$ \\
$42-47$ & $6.0 \pm 0.3$ & $0.54 \pm 0.07$ & $0.99 \pm 0.05$ \\
\hline
\end{tabular}

Notes. ${ }^{(a)}$ Sonnentrucker et al. (2010); ${ }^{(b)}$ Indriolo et al. (2015).

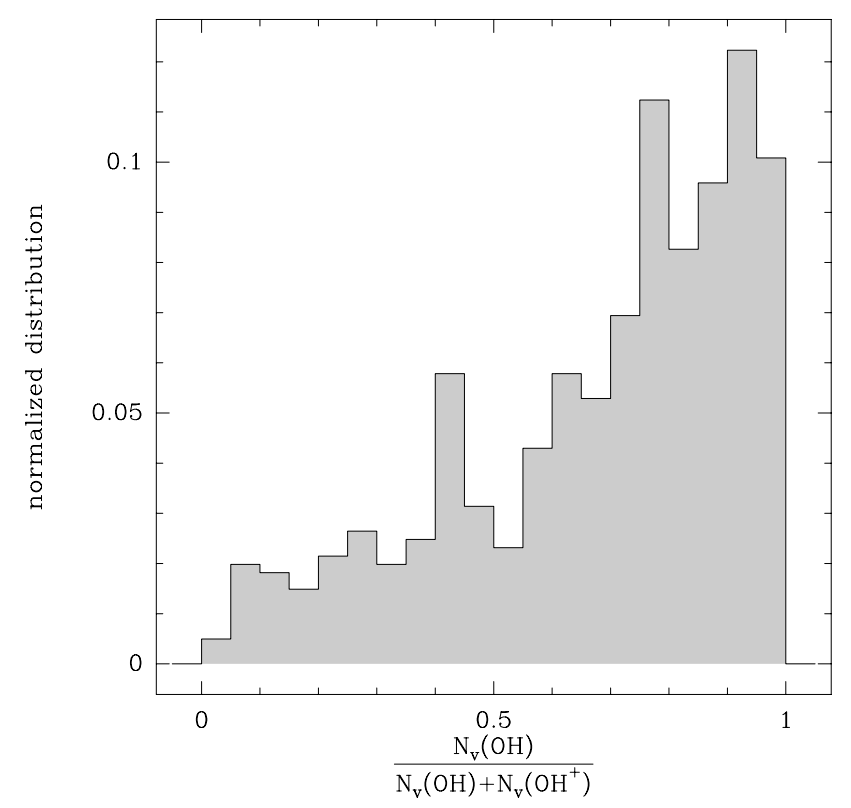

Fig. 12. Normalized distribution of the velocity-specific column density of $\mathrm{OH}, N_{\mathrm{v}}(\mathrm{OH})$, with respect to $N_{\mathrm{v}}(\mathrm{OH})+N_{\mathrm{v}}\left(\mathrm{OH}^{+}\right)$.

\subsection{Gas dynamics and Galactic structure}

In the remainder of this section we briefly address aspects of Galactic structure and gas dynamics. If distance ambiguities at a given velocity can be ruled out for example by measurements of maser parallaxes in the continuum sources (Reid et al. 2014), it is possible to localize the absorbing spiral arm. The absorption may be caused by a single cloud, but more likely by a blend of several clouds. We note that the absorption features seen in $\mathrm{OH}$ tend to be narrower than those observed in $\mathrm{OH}^{+}$, as shown in the distribution of the respective line widths (Fig. 9). The narrowest line widths may thus be due to absorption in a single cloud. In the more likely case of a blend of several clouds the largest line widths are due to large velocity gradients occurring in certain spiral arm crossings. Mild shocks dissipating interstellar turbulence, with slow to moderate velocities (Lesaffre et al. 2013), may also produce large line widths. The distribution of absorption line widths indeed suggests a tail above $\sim 10 \mathrm{~km} \mathrm{~s}^{-1}$, with the bulk of fitted profiles at lower widths. MHD simulations show that the velocity dispersion along the line of sight increases with column density, both in super- and sub-Alfvénic models, but the correlation between these two quantities is stronger in the supersonic than in the subsonic regime (Burkhart et al. 2009; see also Padoan et al. 1998). For $\mathrm{OH}^{+}$the correlation coefficient 
H. Wiesemeyer et al.: Far-infrared study of tracers of oxygen chemistry in diffuse clouds

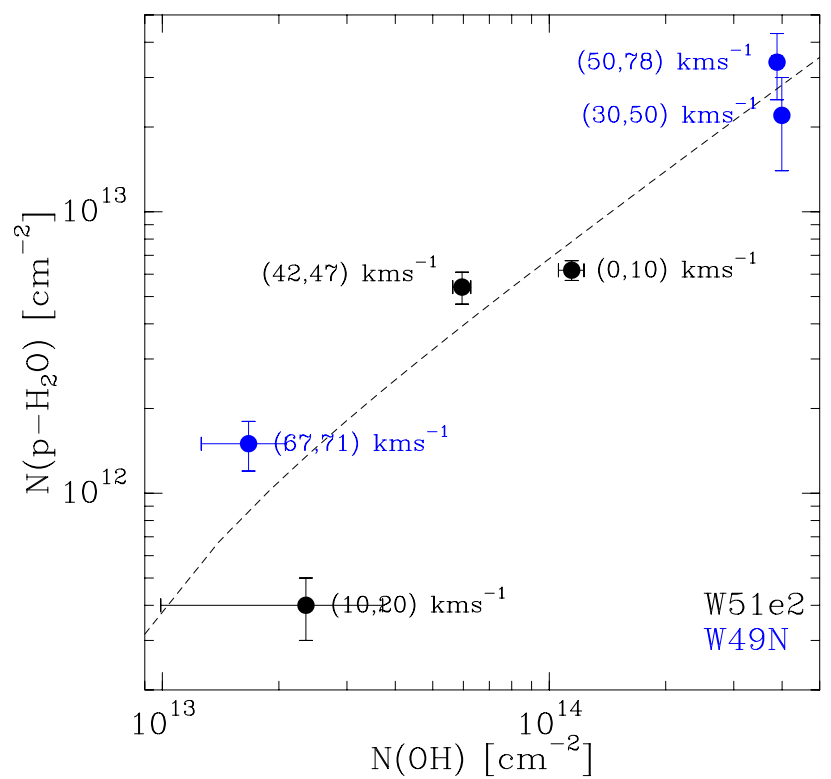

Fig. 13. Correlation between the column densities of $\mathrm{OH}$ (this work) and $p-\mathrm{H}_{2} \mathrm{O}$ (Sonnentrucker et al. 2010), for clouds on the sightlines to W51e2 (black) and W49N (blue). The dashed line shows the linear regression between these quantities. The velocity intervals used to derive the column densities are indicated. $N\left(\mathrm{H}_{2} \mathrm{O}\right)$ values are from Sonnentrucker et al. (2010) and Sonnentrucker et al. (2015).

is 0.35 and the correlation is significant at the $5 \%$ level, that is, $12 \%$ of the variance observed in the line widths is probably due to the expected correlation between column density and line width. For $\mathrm{OH}$, the correlation is much weaker due to the larger number of narrow velocity components, regardless of column density. With a coefficient of 0.16 , no correlation between column density and line width is found with a false-alarm probability of $\leq 5 \%$. Dissipation of turbulence would tend to flatten the correlation. At present we cannot confirm whether the narrow $\mathrm{OH}$ absorption features, originating from diffuse molecular and translucent clouds, are a direct consequence of dissipation of turbulence. However, it seems fair to say that the observed distribution of line widths is a genuine manifestation of the interplay between interstellar turbulence and the association of $\mathrm{OH}^{+}$with more diffuse, atomic and of $\mathrm{OH}$ with denser, molecular gas. The higher contrast of arm to interarm seen in $\mathrm{OH}$ absorption compared to that observed in $\mathrm{OH}^{+}$is yet another manifestation of the anticorrespondence between the two spieces: in Figs. 4 and 5, the groups of enhanced column densitities corresponding to different spiral arm crossings are separated more clearly in $\mathrm{OH}$ than in $\mathrm{OH}^{+}$. A striking example is the sightline to W49N. In the $(20,30) \mathrm{km} \mathrm{s}^{-1}$ interval that we assigned to the interarm region between the Perseus and Sagittarius arms, the ratio between the respective column densities is 5 for $\mathrm{OH}$, but only 2 for $\mathrm{OH}^{+}$. Likewise, Heyer \& Terebey (1998) found for the contrast between the outer Perseus arm and the interarm gas a ratio of 2.5 in atomic gas traced by $\mathrm{HI}$, but a ratio of 28 in molecular gas, traced by CO. We finally note that the characteristic time for crossing a spiral arm, and therefore for the compression of gas by the corresponding density wave, is $\sim 80 \mathrm{Myr}$ in the solar neighborhood, $15 \mathrm{Myr}$ at $R_{\mathrm{G}}=4.5 \mathrm{kpc}$, and $68 \mathrm{Myr}$ at $R_{\mathrm{G}}=7.2 \mathrm{kpc}$, assuming that the diffuse gas rotates with the same drift speed as the stellar clusters for which these crossing times were derived (Gieles et al. 2007). The timescale on which chemical models of diffuse and translucent clouds reach equilibrium abundances is $\sim 10$ Myr (e.g., Heck et al. 1992). It can therefore not be ruled out that the chemistry in the observed clouds, at least for the smaller galactocentric radii, is not in equilibrium. The order-ofmagnitude variation found by us in the fractional abundances of $\mathrm{OH}$ and $\mathrm{OH}^{+}$would agree with this statement, as would the lack of a significant correlation between the $\mathrm{X}(\mathrm{OH}) / \mathrm{X}\left(\mathrm{OH}^{+}\right)$ratio and the molecular hydrogen fraction toward $\mathrm{W} 31 \mathrm{C}$, at low $R_{\mathrm{G}}$ (Fig. 8).

\section{Conclusions}

We presented the first dedicated survey of absorption spectra of $\mathrm{OH}, \mathrm{OH}^{+}$and $\mathrm{OI}$ in diffuse interstellar clouds with adequate velocity resolution, allowing us to separate the observed features into several spiral arm crossings. We conclude with a summary of our most important findings.

1. The OI absorption was observed for three sightlines with unprecedented spectral resolution. We showed that a significant correlation exists between the column density of $\mathrm{O}$ I and that of the total (atomic and molecular) hydrogen reservoir. This is because OI is only slowly removed from the gas phase as diffuse atomic clouds evolve toward diffuse molecular and translucent clouds. We found that the sightlineaveraged $\mathrm{O}$ I abundances toward W31C, G34.26, and W49N are confined to a narrow range of 3.1 to $3.5 \times 10^{-4}$, which agrees reasonably well with earlier measurements (e.g., Lis et al. 2001) and below the abundance of $(5.75 \pm 0.4) \times 10^{-4}$ in the B-type stars of nearby OB-associations (Przybilla et al. 2008). If we take the latter abundance as reference standard, the difference with our abundance measurement can be attributed to the depletion of OI into oxygen-bearing molecules, ices, and dust. However, our result does not exclude the presence of so far unidentified carriers of O I (Jenkins 2009; Whittet 2010). We finally note that with the distance measurements of Reid et al. (2014) and toward suitably chosen sightlines, it should be possible to determine a galactocentric O I abundance gradient from first principles, without the non-LTE modeling required for excited oxygen lines, and free of the opacity limitations of UV spectroscopy.

2. The column densities of $\mathrm{OH}$ are loosely correlated with those of $\mathrm{OH}^{+}$, reflecting the spiral arm crossings on the observed sightlines. The arm-to-interarm contrast is stronger in $\mathrm{OH}$ than in $\mathrm{OH}^{+}$. The two radicals can coexist, but there are more cases where $\mathrm{OH}$ exists without $\mathrm{OH}^{+}$than the other way around. We attribute this finding to the dissociative recombination of $\mathrm{OH}^{+}$occurring if there is not enough $\mathrm{H}_{2}$ to form $\mathrm{OH}$ and $\mathrm{H}_{2} \mathrm{O}$.

3. The $\mathrm{OH} / \mathrm{OH}^{+}$ratio increases as a function of the molecular fraction $f_{\mathrm{H}_{2}}^{\mathrm{N}}=N\left(\mathrm{H}_{2}\right) /\left(N(\mathrm{H})+2 N\left(\mathrm{H}_{2}\right)\right)$. We interpret this as an indication of the importance of the bottleneck reaction $\mathrm{OH}^{+}\left(\mathrm{H}_{2}, \mathrm{H}\right) \mathrm{H}_{2} \mathrm{O}^{+}$for the formation of $\mathrm{OH}$ and $\mathrm{H}_{2} \mathrm{O}$ in cold, ion-neutral driven chemistry, while our $N\left(\mathrm{H}_{2} \mathrm{O}\right) / N(\mathrm{OH})$ ratios do not rule out the endothermic reaction pathways of warm chemistry in turbulent dissipation regions and slow shocks.

4. We estimated the branching ratio for the dissociative recombination of $\mathrm{H}_{3} \mathrm{O}^{+}$into $\mathrm{H}_{2} \mathrm{O}$ and $\mathrm{OH}$ and found that a range of 84 to $91 \%$ of the available $\mathrm{H}_{3} \mathrm{O}^{+}$yields $\mathrm{OH}$. This compares well with laboratory measurements, which yield 74 to $83 \%$.

5. The line widths of the velocity components of the $\mathrm{OH}^{+} \mathrm{ab}-$ sorption are significantly correlated (at the 5\% level) with the column density, as expected from models of MHD turbulence. For $\mathrm{OH}$, there is no such correlation, owing to the frequent occurrence of narrow absorption lines, irrespective 
of column density. It can only be speculated whether the latter finding is to be interpreted as a sign of dissipation of turbulence.

The uncertainties in our analysis are mainly due to velocity crowding on the sightline and to uncertainties regarding the abundances of the $\mathrm{H}_{2}$ tracers. Only the ability of GREAT to observe the ground-state absorption of $\mathrm{OH}$ and $\mathrm{O} \mathrm{I}$ at the required spectral resolution and at frequencies above those covered by HIFI has allowed us to reduce the sightline confusion to an unavoidable minimum and to determine column densities from first principles. In summary, it seems fair to say that our findings further constrain the importance of cold ion-neutral chemistry in the diffuse interstellar medium of the Galaxy without excluding endothermic neutral-neutral and grain surface reactions.

Acknowledgements. Based in part on observations made with the NASA/DLR Stratospheric Observatory for Infrared Astronomy. SOFIA Science Mission Operations are conducted jointly by the Universities Space Research Association, Inc., under NASA contract NAS2-97001, and the Deutsches SOFIA Institut under DLR contract 50 OK 0901 . We GREATfully acknowledge the support by the observatory staff and the careful examination of this work by an anonymous referee. The kinetic data used in our study were downloaded from the online database KIDA (http://kida.obs.u-bordeaux1.fr).

\section{References}

Albertsson, T., Indriolo, N., Kreckel, H., et al. 2014, ApJ, 787, 44 Asplund, M., Grevesse, N., Sauval, A. J., \& Scott, P. 2009, ARA\&A, 47, 481 Bartkiewicz, A., Szymczak, M., \& van Langevelde, H. J. 2014, A\&A, 564, A110 Boreiko, R. T., \& Betz, A. L. 1996, ApJ, 464, L83

Brand, J., \& Blitz, L. 1993, A\&A, 275, 67

Büchel, D., Pütz, P., Jacobs, K., et al. 2015, IEEE Transactions on Terahertz Science and Technology, 5, 207

Burkhart, B., Falceta-Gonçalves, D., Kowal, G., \& Lazarian, A. 2009, ApJ, 693, 250

Cartledge, S. I. B., Lauroesch, J. T., Meyer, D. M., \& Sofia, U. J. 2004, ApJ, 613, 1037

Caswell, J. L. 1998, MNRAS, 297, 215

Caswell, J. L., Vaile, R. A., Ellingsen, S. P., Whiteoak, J. B., \& Norris, R. P. 1995, MNRAS, 272, 96

Comito, C., Schilke, P., Phillips, T. G., et al. 2005, ApJS, 156, 127

Cowley, C. R. 1995, An Introduction to Cosmochemistry (Cambridge University Press), 502

Cox, D. P. 2005, ARA\&A, 43, 337

Csengeri, T., Menten, K. M., Wyrowski, F., et al. 2012, A\&A, 542, L8

Csengeri, T., Urquhart, J. S., Schuller, F., et al. 2014, A\&A, 565, A75

de Graauw, T., Helmich, F. P., Phillips, T. G., et al. 2010, A\&A, 518, L6

Deshpande, A. A., Goss, W. M., \& Mendoza-Torres, J. E. 2013, ApJ, 775, 36

Draine, B. T. 1978, ApJS, 36, 595

Draine, B. T. 2011, Physics of the Interstellar and Intergalactic Medium (Princeton University Press)

Dunham, T., Jr. 1937, PASP, 49, 26

Elitzur, M., \& Asensio Ramos, A. 2006, MNRAS, 365, 779

Emprechtinger, M., Monje, R. R., van der Tak, F. F. S., et al. 2012, ApJ, 756, 136 Esteban, C., García-Rojas, J., Peimbert, M., et al. 2005, ApJ, 618, L95

Fish, V. L., Reid, M. J., Argon, A. L., \& Zheng, X.-W. 2005, ApJS, 160, 220

Flagey, N., Goldsmith, P. F., Lis, D. C., et al. 2013, ApJ, 762, 11

Gerin, M., de Luca, M., Goicoechea, J. R., et al. 2010a, A\&A, 521, L16

Gerin, M., de Luca, M., Black, J., et al. 2010b, A\&A, 518, L110

Gieles, M., Athanassoula, E., \& Portegies Zwart, S. F. 2007, MNRAS, 376, 809

Godard, B., Falgarone, E., \& Pineau Des Forêts, G. 2009, A\&A, 495, 847

Godard, B., Falgarone, E., Gerin, M., et al. 2012, A\&A, 540, A87

Gómez, G. C., Benjamin, R. A., \& Cox, D. P. 2001, AJ, 122, 908

Green, J. A., \& McClure-Griffiths, N. M. 2011, MNRAS, 417, 2500

Guan, X., Stutzki, J., Graf, U. U., et al. 2012, A\&A, 542, L4

Hafok, H., \& Polehampton, E. 2013, Apex Calibration and Data Reduction

Manual, rev. 1.2.1

Heck, E. L., Flower, D. R., Le Bourlot, J., Pineau des Forets, G., \& Roueff, E. 1992, MNRAS, 258, 377

Heyer, M. H., \& Terebey, S. 1998, ApJ, 502, 265

Heyminck, S., Graf, U. U., Güsten, R., et al. 2012, A\&A, 542, L1
Hollenbach, D. J., Takahashi, T., \& Tielens, A. G. G. M. 1991, ApJ, 377, 192 Hollenbach, D., Kaufman, M. J., Neufeld, D., Wolfire, M., \& Goicoechea, J. R. 2012, ApJ, 754, 105

Indriolo, N., Neufeld, D. A., Seifahrt, A., \& Richter, M. J. 2013, ApJ, 764, 188

Indriolo, N., Neufeld, D. A., Gerin, M., et al. 2015, ApJ, 800, 40

Jenkins, E. B. 2009, ApJ, 700, 1299

Jensen, M. J., Bilodeau, R. C., Safvan, C. P., et al. 2000, ApJ, 543, 764

Jensen, A. G., Rachford, B. L., \& Snow, T. P. 2005, ApJ, 619, 891

Jönsson, H., Ryde, N., Harper, G. M., Richter, M. J., \& Hinkle, K. H. 2014, ApJ, 789, L41

Kafle, P. R., Sharma, S., Lewis, G. F., \& Bland-Hawthorn, J. 2014, ApJ, 794, 59

Klein, B., Hochgürtel, S., Krämer, I., et al. 2012, A\&A, 542, L3

Korotin, S. A., Andrievsky, S. M., Luck, R. E., et al. 2014, MNRAS, 444, 3301

Krełowski, J., Beletsky, Y., \& Galazutdinov, G. A. 2010, ApJ, 719, L20

Kurayama, T., Nakagawa, A., Sawada-Satoh, S., et al. 2011, PASJ, 63, 513

Lallement, R. 2007, Space Sci. Rev., 130, 341

Leinz, C., Caris, M., Klein, T., et al. 2010, Proc. Twenty-First Int. Symp. Space Terahertz Technol., 130

Le Petit, F., Nehmé, C., Le Bourlot, J., \& Roueff, E. 2006, ApJS, 164, 506

Lesaffre, P., Pineau des Forêts, G., Godard, B., et al. 2013, A\&A, 550, A106

Lis, D. C., Keene, J., Phillips, T. G., et al. 2001, ApJ, 561, 823

Liszt, H., \& Lucas, R. 2002, A\&A, 391, 693

Maret, S. 2014, Astrochem. http://smaret.github.io/astrochem/

Mathis, J. S., Mezger, P. G., \& Panagia, N. 1983, A\&A, 128, 212

Melnick, G. J., \& Bergin, E. A. 2005, Adv. Space Res., 36, 1027

Melnick, G., Gull, G. E., \& Harwit, M. 1979, ApJ, 227, L29

Metropolis, N., Rosenbluth, A. W., Rosenbluth, M. N., Teller, A. H., \& Teller, E. 1953, J. Chem. Phys., 21, 1087

Meyer, D. M., Jura, M., \& Cardelli, J. A. 1998, ApJ, 493, 222

Müller, H. S. P., Thorwirth, S., Roth, D. A., \& Winnewisser, G. 2001, A\&A, 370 L49

Neufeld, D. A., \& Wolfire, M. G. 2009, ApJ, 706, 1594

Neufeld, D. A., Kaufman, M. J., Goldsmith, P. F., Hollenbach, D. J., \& Plume, R. 2002, ApJ, 580, 278

Neufeld, D. A., Goicoechea, J. R., Sonnentrucker, P., et al. 2010, A\&A, 521, L10

Neau, A., Al Khalili, A., Rosén, S., et al. 2000, J. Chem. Phys., 113, 1762

Padoan, P., Juvela, M., Bally, J., \& Nordlund, A. 1998, ApJ, 504, 300

Pagel, B. E. J. 1973, Cosmochemistry, 40, 1

Pickett, H. M., Poynter, R. L., Cohen, E. A., et al. 1998, JQSRT, 60, 883

Poglitsch, A., Waelkens, C., Geis, N., et al. 2010, A\&A, 518, L2

Press, W. H., Teukolsky, S. A., Vetterling, W. T., \& Flannery, B. P. 1992 Numerical Recipes in $\mathrm{C}$ : the Art of Scientific Computing (Cambridge: University Press)

Przybilla, N., Nieva, M.-F., \& Butler, K. 2008, ApJ, 688, L103

Qiu, K., Wyrowski, F., Menten, K. M., et al. 2011, ApJ, 743, L25

Reid, M. J., Menten, K. M., Brunthaler, A., et al. 2014, ApJ, 783, 130

Richter, H., Wienold, M., Schrottke, L., et al. 2015, IEEE Transations on Terahertz Science and Technology, submitted

Sanna, A., Reid, M. J., Menten, K. M., et al. 2014, ApJ, 781, 108

Sato, M., Reid, M. J., Brunthaler, A., \& Menten, K. M. 2010, ApJ, 720, 1055

Sheffer, Y., Rogers, M., Federman, S. R., et al. 2008, ApJ, 687, 1075

Smartt, S. J., \& Rolleston, W. R. J. 1997, ApJ, 481, L47

Snow, T. P., \& McCall, B. J. 2006, ARA\&A, 44, 367

Sodroski, T. J., Odegard, N., Arendt, R. G., et al. 1997, ApJ, 480, 173

Sonnentrucker, P., Neufeld, D. A., Phillips, T. G., et al. 2010, A\&A, 521, L12

Sonnentrucker, P., Wolfire, M., Neufeld, D. A., et al. 2015, ApJ, 806, 49

Sormani, M. C., \& Magorrian, J. 2015, MNRAS, 446, 4186

Surcis, G., Vlemmings, W. H. T., van Langevelde, H. J., \& Hutawarakorn

Kramer, B. 2012, A\&A, 541, A47

Swings, P., \& Rosenfeld, L. 1937, ApJ, 86, 483

Tizniti, M., Le Picard, S. D., Lique, F., et al. 2014, Nat. Chem., 6, 141

Vallée, J. P. 2008, AJ, 135, 1301

van Dishoeck, E. F. 1998, The Molecular Astrophysics of Stars and Galaxies, eds. T. W. Hartquist, \& D. A. Williams (Oxford: Clarendon Press), 4, 53

van Dishoeck, E. F., \& Black, J. H. 1986, ApJS, 62, 109

Vasyunin, A. I., Semenov, D. A., Wiebe, D. S., \& Henning, T. 2009, ApJ, 691, 1459

Voshchinnikov, N. V., Semenov, D. A., \& Henning, T. 1999, A\&A, 349, L25

Wakelam, V., \& KIDA Team 2012, EAS Publ. Ser., 58, 287

Whittet, D. C. B. 2010, ApJ, 710, 1009

Wienen, M., Wyrowski, F., Menten, K. M., et al. 2015, A\&A, 579, A91

Wiesemeyer, H., Güsten, R., Heyminck, S., et al. 2012, A\&A, 542, L7

Wyrowski, F., Menten, K. M., Güsten, R., \& Belloche, A. 2010, A\&A, 518, A26

Zhang, B., Reid, M. J., Menten, K. M., et al. 2013, ApJ, 775, 79 


\section{Appendix A: Comments on individual sightlines}

The next two paragraphs introduce the observed sightlines, that is, their spiral arm crossing, and provide a phenomenological description of the absorption spectra.

\section{A.1. First quadrant}

G10.47 - The sightline toward G10.47, located at $8.5 \mathrm{kpc}$ in the connecting arm (Sanna et al. 2014), exhibits a relatively broad absorption spectrum. The features are from the near side of the Sagittarius arm, the Scutum arm, the near $3 \mathrm{kpc}$ arm, and the Galactic bar. By consequence, the column density profiles of $\mathrm{OH}$ and $\mathrm{OH}^{+}$extend from -20 to $160 \mathrm{~km} \mathrm{~s}^{-1}$ with a rough correspondence between $\mathrm{OH}$ and $\mathrm{OH}^{+}$and generally narrower features in $\mathrm{OH}$. There are three major components, at velocities redshifted with respect to the hot core. They probably belong to the $135 \mathrm{~km} \mathrm{~s}^{-1}$ arm (Sormani \& Magorrian 2015, further references therein).

G10.62 (W31C) - G10.62 is located at a distance of $4.95 \mathrm{kpc}$ in the $3 \mathrm{kpc}$ arm (Sanna et al. 2014). The sightline to it crosses the near sides of the Sagittarius and Scutum arms, with projected velocities ranging from 0 to $50 \mathrm{~km} \mathrm{~s}^{-1}$. The column densities of $\mathrm{OH}$ and $\mathrm{OH}^{+}$are roughly correlated with each other, except for an $\mathrm{OH}^{+}$feature at 60 to $80 \mathrm{~km} \mathrm{~s}^{-1}$ without a corresponding $\mathrm{OH}$ feature. Likewise, another local maximum of the $\mathrm{OH}^{+}$column density at about $10 \mathrm{~km} \mathrm{~s}^{-1}$ is not associated with a corresponding maximum in the distribution of $\mathrm{OH}$ features and represents another remarkable example of an anticorrespondence of the two species.

G34.26-G34.26 is located in the Sagittarius spiral arm, which is the only one crossed by this sightline. Its distance of $1.56 \mathrm{kpc}$ is based on the assumption that $\mathrm{G} 34.34\left(\mathrm{H}_{2} \mathrm{O}\right.$ maser parallax, Kurayama et al. 2011) belongs to the same complex. $\mathrm{OH}$ is seen from 45 to $60 \mathrm{~km} \mathrm{~s}^{-1}$ and, after a local mininum, from 60 to $75 \mathrm{~km} \mathrm{~s}^{-1} \cdot \mathrm{OH}^{+}$is only observed in the first interval. Another group of $\mathrm{OH}$ at 0 to $15 \mathrm{~km} \mathrm{~s}^{-1}$ is framed by local maxima of $\mathrm{OH}^{+}$, again in anticorrelation. Interestingly, $\mathrm{OH}^{+}$is seen along the whole sightline, that is, in the interval from -5 to $+75 \mathrm{~km} \mathrm{~s}^{-1}$.

W49N - W49N (11.1 kpc distance, Zhang et al. 2013) shows absorption in three groups, whose separation is clearer in $\mathrm{OH}$ than in $\mathrm{OH}^{+}$. The three groups with velocities from 30 to $70 \mathrm{~km} \mathrm{~s}^{-1}$ arise from a sightline grazing the Sagittarius arm, while the gas from -10 to $20 \mathrm{~km} \mathrm{~s}^{-1}$ is located in the Perseus arm in which W49 is located. Between these two velocity intervals, $\mathrm{OH}^{+}$is seen with much less contrast (with respect to the column densities in the two velocity intervals) than $\mathrm{OH}$, which leads us to assume that the interval from 20 to $30 \mathrm{~km} \mathrm{~s}^{-1}$ contains interarm gas located close to the Perseus arm.

W51e2 - The sightline to W51e2, at $5.4 \mathrm{kpc}$ distance (Sato et al. 2010) closer than W49N but at similar Galactic longitude, tangentially follows the Sagittarius arm, with velocities from 40 to $75 \mathrm{~km} \mathrm{~s}^{-1}$ and in the 0 to $10 \mathrm{~km} \mathrm{~s}^{-1}$ interval. Between these velocity intervals we detected no $\mathrm{OH}$ but $\mathrm{OH}^{+}$.

\section{A.2. Fourth quadrant}

G327.29 - For this hot core Green \& McClure-Griffiths (2011) provided no distance estimate. The in projection nearby
G329.031 (like G327.29 below the Galactic plane) was shown to be at $3.2 \mathrm{kpc}$ distance. This would place the target in the Crux spiral arm, in agreement with the velocities of its associated $\mathrm{OH}$ and $\mathrm{CH}_{3} \mathrm{OH}$ masers. Wienen et al. (2015) determined the distance to $3.1 \mathrm{kpc}$, using the rotation curve of Brand \& Blitz (1993) and solving the distance ambiguity by means of H I selfabsorption. The $\mathrm{OH}$ column in the $(-10 \pm 10) \mathrm{km} \mathrm{s}^{-1}$ interval coincides with the crossing of the Carina arm, the first one on the sightline from the observer to G327.29. The velocity distributions of $\mathrm{OH}$ and $\mathrm{OH}^{+}$are correlated, but with markedly different widths.

G330.95 - Its distance of $4.7 \mathrm{kpc}$ (Green \& McClure-Griffiths 2011) to $5.7 \mathrm{kpc}$ (Wienen et al. 2015) locates this target in the Norma spiral arm. The Carina and Crux arms are traced at about $0 \mathrm{~km} \mathrm{~s}^{-1}$ and in the $(-50 \pm 10) \mathrm{km} \mathrm{s}^{-1}$ interval, respectively. The absorption in the interval at $(-90 \pm 10) \mathrm{km} \mathrm{s}^{-1}$ is from the Norma arm and the environment of the hot core. The much wider distribution of $\mathrm{OH}^{+}$is only loosely correlated with that of $\mathrm{OH}$. Notably, the crossing of the Carina arm only shows in $\mathrm{OH}^{+}$absorption, but not in $\mathrm{OH}$.

G332.83 - The distance to G332.83 is undetermined in Green $\&$ McClure-Griffiths (2011). The in projection nearby region G332.81 is located at $11.7 \mathrm{kpc}$ distance in the far side of the Crux spiral arm. If we were to adopt this distance, the sightline would cross the same spiral arms as G330.95 but twice the Crux arm: The first crossing is at $\sim 4 \mathrm{kpc}$, the second one close to the target itself. However, the resulting tangential crossing of the Norma arm at $\sim 100 \mathrm{~km} \mathrm{~s}^{-1}$, see also Vallée (2008) (his Fig. 2) leaves no trace of an absorption, neither in $\mathrm{OH}$ nor in $\mathrm{OH}^{+}$. We use this as an indication that G332.83 is located in the near side of the Crux arm. Interestingly, Wienen et al. (2015) also adopted the near-side distance of $3.2 \mathrm{kpc}$, based on a spectrophotometric measurement.

G351.58 - At $5.1 \mathrm{kpc}$ distance (Green \& McClure-Griffiths 2011), the hot core is located near the beginning of the Norma or in the near $3 \mathrm{kpc}$ arm; this is also evidenced by its high negative systemic velocity. Wienen et al. (2015) corrected this distance to a value of $6.8 \mathrm{kpc}$ (with HI self-absorption). As for the other sightlines in the fourth quadrant, the gas near zero velocities stems from the Carina arm, while the group ranging from -70 to $-40 \mathrm{~km} \mathrm{~s}^{-1}$ belongs to the Crux arm, which is crossed over a length of $\sim 1.5 \mathrm{kpc}$. The gap until the $(-100,-90) \mathrm{km} \mathrm{s}^{-1}$ interval is filled by interarm gas seen in $\mathrm{OH}^{+}$with a broad velocity distribution, but not by a measurable column density of $\mathrm{OH}$.

\section{Appendix B: Observational bias in abundance averages}

As previously emphasized, absorption spectroscopy against spatially unresolved background continuum sources samples the column density, and not the mean gas density. Consequently, the determination of relevant abundances, or abundance ratios, is not straightforward. If such quantities are expected to qualify for conclusions regarding a given spiral arm crossing, the question of a potential observational bias has to be addressed. Usually, column density averages across the corresponding velocity interval $\left(v_{1}, v_{2}\right)$ are applied. If $X$ and $Y$ denote the column densities per unit velocity interval of the two species to be compared, this leads to ratios $r$ like

$r=\frac{\int_{v_{1}}^{v_{2}} X(v) \mathrm{d} v}{\int_{v_{1}}^{v_{2}} Y(v) \mathrm{d} v}$. 
The following analysis shows that in practice such ratios are only of limited use if observational bias is to be avoided. We note that an absorption line profile is mainly decomposed for deconvolution from hyperfine splitting (and for the sake of internal consistency also for species without hyperfine splitting), but not to identify individual Gaussian components as single, veritable diffuse clouds. Considering therefore that each velocity within the interval $\left(v_{1}, v_{2}\right)$ represents a different ensemble of diffuse cloud entities, ratios like

$r=\int_{v_{1}}^{v_{2}} \frac{X(v)}{Y(v)} w(v) \mathrm{d} v$

are preferred, where $w(v)$ is a normalized weight function. The most appropriate choice, namely by mass per unit velocity interval, is also the most inaccessible one, especially in absorption spectroscopy toward unresolved continuum sources in the background. The ratios defined in Eqs. (B.1) and (B.2) are equal only if the weight function is defined through the column density of the reference species, that is,

$w(v)=\frac{Y(v)}{\int_{v_{1}}^{v_{2}} Y(\tilde{v}) \mathrm{d} \tilde{v}}$.

For the most frequently encountered case, that is, for a diffuse cloud whose angular extent exceeds that of the unresolved background source, such a weighting would depend on the distance $D$ of the absorbing cloud: Assuming that a typical Galactic hot core extends over a typical size of $L_{\mathrm{hc}} \sim 10^{-2}$ to $10^{-1} \mathrm{pc}$ and a diffuse cloud over a typical scale of $L_{\mathrm{dc}} \sim 10 \mathrm{pc}$, absorption spectroscopy of a diffuse cloud (at distance $D_{\mathrm{dc}}$ ) against a spatially unresolved continuum source at distance $D_{\mathrm{hc}}$ traces a cloud volume fraction of

$f_{\mathrm{DC}}^{(\mathrm{V})} \sim\left(\frac{L_{\mathrm{hc}}}{L_{\mathrm{dc}} D_{\mathrm{hc}}}\right)^{2} D_{\mathrm{dc}}^{2}$.

If we consider the diffuse cloud as a whole, only a volume fraction of at most $\sim 0.01 \%$ is traced at any distance, which is not necessarily representative for the entire cloud. However, it is conceivable that what is observed as a single diffuse cloud consists of a blend of several cloudlets of a typical size of $\sim 1 \mathrm{pc}$, possibly showing abundance variations among them (Lallement 2007, further references therein) The measured subvolume now amounts to at most $1 \%$ for a cloudlet close to the hot core, but decreases to $0.25 \%$ for a cloudlet located half-way to the hot core, leading to a corresponding weighting of column densities although both cloudlets are equally representative of the abundance study. Such a situation naturally arises due to the velocity amibiguity encountered in near- and far-side crossings of a given spiral arm. Moreover, the deduced column densities are by no means representative of the total mass of clouds at a given lineof-sight velocity. We therefore prefer Eq. (B.2), with a uniform weight function, $w(v) \equiv 1$ in the velocity interval $\left(v_{1}, v_{2}\right)$. The rationale behind this apriority is the assumption that each velocity within an interval corresponding to a given spiral arm crossing is equally representative for the analysis of the chemistry in diffuse clouds. This weighting requires elimination of velocity components with an insignificant $Y(v)$, in order to avoid divergence. Here we used a conservative cutoff of $5 \sigma_{\text {rms }}$. This parameter was varied so as to ensure that the derived abundance ratios did not vary by more than their inherent mainly noise-induced uncertainty.
Table C.1. Rate coefficients for the reactions considered in this work (from Wakelam et al. 2012 for $\mathrm{H}_{2}$ abstraction reactions, and from Hollenbach et al. 2012 for photoionization and photodissociation).

\begin{tabular}{|c|c|c|}
\hline \multirow{4}{*}{$\begin{array}{l}\mathrm{OH}^{+}\left(\mathrm{e}^{-}, \mathrm{H}\right) \mathrm{O} \\
\mathrm{H}_{3} \mathrm{O}^{+}\left(\mathrm{e}^{-}, \mathrm{H}\right) \mathrm{OH} \\
\mathrm{H}_{3} \mathrm{O}^{+}\left(\mathrm{e}^{-}, \mathrm{H}_{2}\right) \mathrm{H}_{2} \mathrm{O}\end{array}$} & \multicolumn{2}{|c|}{$\begin{array}{l}\text { Dissociative recombination rates } \\
\text { model } 1(100 \mathrm{~K}) \quad \text { model } 2(15 \mathrm{~K}) \\
{\left[\mathrm{cm}^{3} \mathrm{~s}^{-1}\right]}\end{array}$} \\
\hline & $6.58 \times 10^{-8}$ & $1.47 \times 10^{-8}$ \\
\hline & $5.54 \times 10^{-7}$ & $1.43 \times 10^{-6}$ \\
\hline & $1.91 \times 10^{-7}$ & $4.92 \times 10^{-7}$ \\
\hline & \multicolumn{2}{|c|}{$\begin{array}{c}\mathrm{H}_{2} \text { abstraction reactions } \\
{\left[\mathrm{cm}^{3} \mathrm{~s}^{-1}\right]}\end{array}$} \\
\hline $\begin{array}{l}\mathrm{OH}^{+}\left(\mathrm{H}_{2}, \mathrm{H}\right) \mathrm{H}_{2} \mathrm{O}^{+} \\
\mathrm{H}_{2} \mathrm{O}^{+}\left(\mathrm{H}_{2}, \mathrm{H}\right) \mathrm{H}_{3} \mathrm{O}^{+}\end{array}$ & \multicolumn{2}{|c|}{$\begin{array}{l}1.1 \times 10^{-9} \\
6.1 \times 10^{-10}\end{array}$} \\
\hline & \multicolumn{2}{|c|}{$\begin{array}{c}\text { Photodissociation rates }(\chi=1.7 \text { Habing }) \\
\text { model } 1\left(A_{\mathrm{V}}=0.2\right) \text { model } 2\left(A_{\mathrm{V}}=1.0\right) \\
{\left[\mathrm{s}^{-1}\right]}\end{array}$} \\
\hline $\mathrm{H}_{2} \mathrm{O}(h v, \mathrm{H}) \mathrm{OH}$ & $5.3 \times 10^{-10}$ & $1.4 \times 10^{-10}$ \\
\hline $\mathrm{H}_{2} \mathrm{O}\left(h v, \mathrm{H}_{2}\right) \mathrm{O}$ & $3.1 \times 10^{-11}$ & $5.3 \times 10^{-12}$ \\
\hline \multirow[t]{2}{*}{$\mathrm{OH}(h v, \mathrm{H}) \mathrm{O}$} & $2.8 \times 10^{-10}$ & $7.1 \times 10^{-11}$ \\
\hline & \multicolumn{2}{|c|}{$\begin{array}{c}\text { Photoionization rates }(\chi=1.7 \text { Habing }) \\
\text { model } 1\left(A_{\mathrm{V}}=0.2\right) \text { model } 2\left(A_{\mathrm{V}}=1.0\right) \\
{\left[\mathrm{s}^{-1}\right]}\end{array}$} \\
\hline $\mathrm{H}_{2} \mathrm{O}\left(h v, \mathrm{e}^{-} e\right) \mathrm{H}_{2} \mathrm{O}^{+}$ & $1.4 \times 10^{-11}$ & $6.3 \times 10^{-13}$ \\
\hline $\mathrm{OH}\left(h v, \mathrm{e}^{-}\right) \mathrm{OH}^{+}$ & $9.8 \times 10^{-12}$ & $3.8 \times 10^{-13}$ \\
\hline
\end{tabular}

\section{Appendix C: Rates of dissociative recombinations, hydrogen abstraction, and photoionization}

The production rate for a chemical reaction $\mathrm{A}+\mathrm{B} \rightarrow \mathrm{C}+\mathrm{D}$ with activation energy $\epsilon_{0}$ is given by (e.g., Cowley 1995)

$n(\mathrm{~A}) n(\mathrm{~B}) k(\epsilon)=n(\mathrm{~A}) n(\mathrm{~B}) \int_{\epsilon_{0}}^{\infty} \sigma(\epsilon) \Phi(\epsilon) \mathrm{d} \epsilon$

where $\sigma(\epsilon)$ is the cross section and $\Phi(\epsilon)$ the distribution of the relative energy $\epsilon$ of species A and B. The parametrization of $\sigma(\epsilon)$ (i.e., the steepness of the energy barrier) leads to the widely used expression

$k(T)=\alpha\left(\frac{T}{300}\right)^{\beta} \exp \left(-\frac{\gamma}{T}\right)$.

For the reaction chain of hydrogen abstractions $\mathrm{OH}^{+}\left(\mathrm{H}_{2}, \mathrm{H}\right) \mathrm{H}_{2} \mathrm{O}^{+}\left(\mathrm{H}_{2}, \mathrm{H}\right) \mathrm{H}_{3} \mathrm{O}^{+}$the parameters $\alpha, \beta$, and $\gamma$ are calculated by means of the Kinetic Database for Astrochemistry (Wakelam et al. 2012) for the conditions defined in Table 3. The resulting reaction rates are summarized in Table C.1. The rate coefficients of the two dissociative recombinations differ by a factor 1.8, independently of temperature (within the range under consideration). Without a chemical model at hand (see Appendix D), the actual production rates are difficult to estimate. The measured abundance ratio $X\left(\mathrm{OH}^{+}\right) / X\left(\mathrm{H}_{2} \mathrm{O}^{+}\right)$of up to 10 (Indriolo et al. 2015) reflects the result of this chemistry rather than its initial conditions. The by three orders of magnitude larger rate coefficient for the dissociative recombination of $\mathrm{H}_{3} \mathrm{O}^{+}$is due to the large cross section for this reaction and does not imply a correspondingly higher production rate of $\mathrm{H}_{2} \mathrm{O}$ and $\mathrm{OH}$, given the fraction of free electrons (typically $10^{-4}$ in diffuse atomic and molecular gas, and $10^{-5}$ and less in translucent clouds where it is maintained by cosmic ray ionization). 

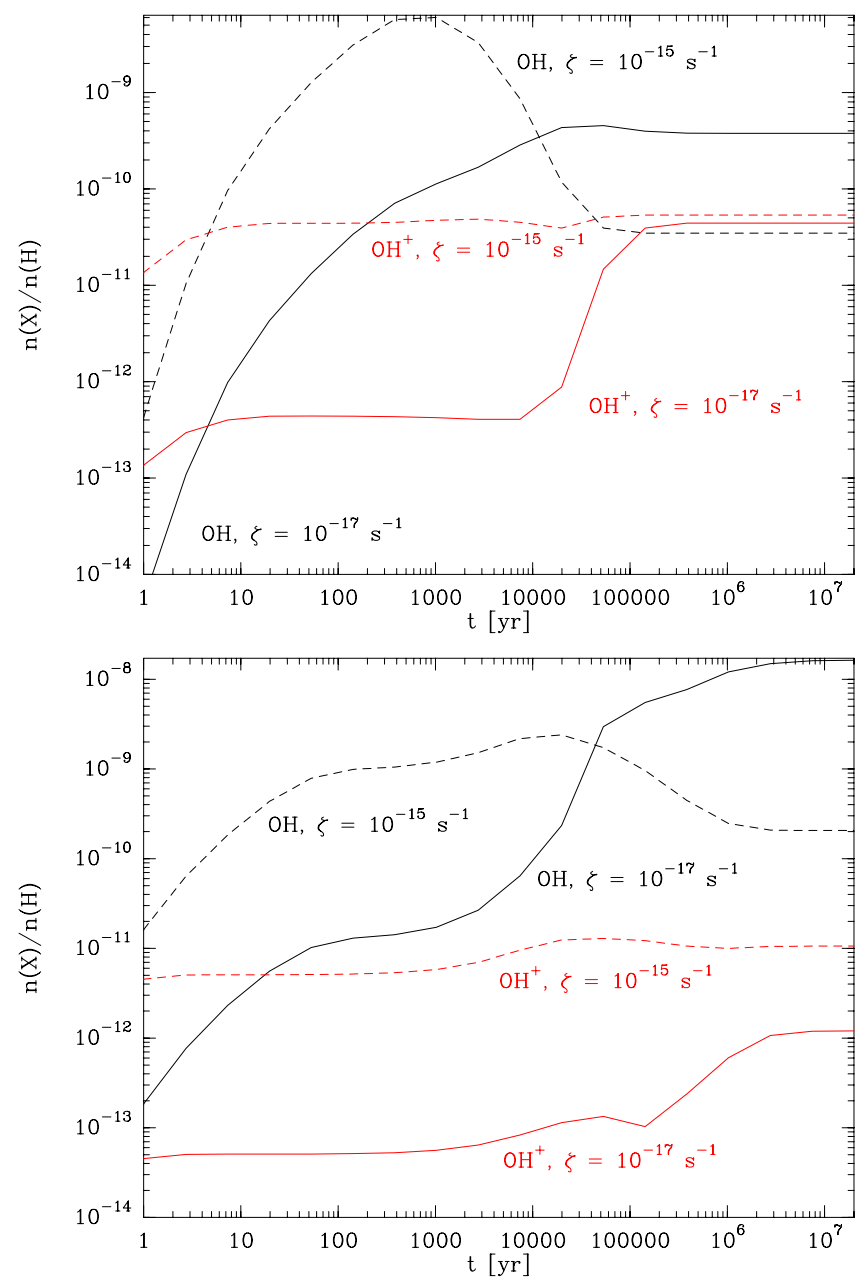

Fig. D.1. Chemical evolution for conditions typical of diffuse molecular (model 1, top) and translucent clouds (model 2, bottom). For details see text. Dashed curves refer to the case with enhanced cosmic ionization rate $\zeta=10^{-15} \mathrm{~s}^{-1}$.

\section{Appendix D: Simple single-layer chemical model}

To estimate the production rate of $\mathrm{OH}$ and $\mathrm{OH}^{+}$we used a simple single-layer model. The purpose of this demonstration is not a quantitative analysis, which would require dedicated PDR and TDR models (Hollenbach et al. 1991; Godard et al. 2009, respectively). We used the Astrochem $\operatorname{code}^{8}$ (Maret 2014) together with the reaction coefficients compiled by the KIDA database $^{9}$ (Wakelam et al. 2012). The models are defined in Table 3. We used two values for the rate of ionization by cosmic rays, $\zeta=10^{-15}$ and $10^{-17} \mathrm{~s}^{-1}$, and assumed the interstellar radiation field to be characterized by a typical value of 1.7 Habing fields. The initial abundances with respect to $\mathrm{H}$ are 0.4 for $\mathrm{H}_{2}$, 0.14 for $\mathrm{He}$, and $3.2 \times 10^{-4}$ for $\mathrm{O} \mathrm{I}$.

The results are shown in Fig. D.1. During the evolution toward equilibrium chemistry the $\mathrm{O}$ I abundance remains constant at the initial value, except for the translucent cloud (model 2), where it drops to $2 \times 10^{-4}$ after $\sim 10^{5}$ years because it is used for the enhanced production of $\mathrm{OH}$ and $\mathrm{H}_{2} \mathrm{O}$ thanks to the higher $\mathrm{H}_{2}$ fraction. However, for a translucent cloud exposed to the higher cosmic-ray ionization rate $\left(\zeta=10^{-15}\right)$, the O I reservoir can be replenished by the cosmic-ray-induced dissociation of

\footnotetext{
8 http://smaret.github.io/astrochem/

9 http://kida.obs.u-bordeaux1.fr
}

$\mathrm{OH}$ and $\mathrm{H}_{2} \mathrm{O}$. In this case, the ratio $n(\mathrm{OH}) / n\left(\mathrm{OH}^{+}\right)$can reach values below unity. For translucent clouds with their higher molecular hydrogen fraction, the conversion of $\mathrm{OH}^{+}$to $\mathrm{OH}$ is much faster, as expected, with an $\mathrm{OH} / \mathrm{OH}^{+}$abundance ratio increased by up to several orders of magnitude. When chemical equilibrium is reached, the remaining column density of $\mathrm{OH}^{+}$ is too low to be detectable, leading to the observed peak in the contrast ratio shown in Fig. 12.

While these simple models qualitatively agree with our findings, a more realistic model would include not only a PDR and dissipation of turbulence, but also a model for the evolution from the diffuse atomic gas to a molecular cloud. In this situation, which is beyond the scope of this paper, the chemical equilibrium would be reached much later.

\section{Appendix E: Comparison of abundances of $\mathrm{CH}$ and $\mathrm{HF}$}

To determine fractional abundances, we used $\mathrm{HF}$ and $\mathrm{CH}$ as surrogates for $\mathrm{H}_{2}$ (Neufeld \& Wolfire 2009; Sonnentrucker et al. 2010). The interstellar chemistry of HF involves only four reactions and can form in an exothermic reaction with $\mathrm{H}_{2}$. Godard et al. (2012) predicted an HF abundance of $X(\mathrm{HF}) / X\left(\mathrm{H}_{2}\right)=$ $3.6 \times 10^{-8}$, which is thought to hold for $A_{\mathrm{V}}>0.2$ and $n_{\mathrm{H}}>$ $30 \mathrm{~cm}^{-3}$, but pointed out that this value needs to be confirmed by observations. Furthermore, fluorine forms in AGB stars, at least in the solar neighborhood (Jönsson et al. 2014), while it is destroyed in massive stars. It is therefore possible that fluorine has a Galactic abundance gradient. Indriolo et al. (2013) have measured $X(\mathrm{HF})$ using the $v=1-0, R(0)$ ro-vibrational transition at $\lambda 2.5 \mu \mathrm{m}$ and found values (e.g., $X(\mathrm{HF})=1.15 \times 10^{-8}$ in the diffuse gas toward HD 154368) significantly below the prediction. Sonnentrucker et al. (2015) confirmed these findings with models that yield $X(\mathrm{HF}) \sim 0.9 \times 10^{-8}$ in the lowdensity regime (at $A_{\mathrm{V}} \sim 0.08 \mathrm{mag}$ ) to $X(\mathrm{HF}) \sim 3.3 \times 10^{-8}$ at $A_{\mathrm{V}} \sim 4$ mag. The lower HF abundance results from recent laboratory measurements (Tizniti et al. 2014) that correct the reaction rate $\mathrm{F}\left(\mathrm{H}_{2}, \mathrm{H}\right) \mathrm{HF}$ downward. Using $X(\mathrm{CH})=3.5 \times 10^{-8}$ (Sheffer et al. 2008, UV spectroscopy), Gerin et al. (2010a, further references therein) showed that their $\mathrm{CCH}$ abundance agrees with earlier findings. Emprechtinger et al. (2012) determined $X(\mathrm{HF})=1.5 \times 10^{-8}$ in the diffuse foreground gas toward massive star-forming regions, again using the canonical $\mathrm{CH}$ abundance from Sheffer et al. (2008). We note that these measurements are fraught with uncertainties of typically 30 to $50 \%$. However, the overall agreement, supported by the laboratory measurements, suggests that the $X(\mathrm{CH})=3.5 \times 10^{-8}$ and $X(\mathrm{HF}) / X(\mathrm{CH})=0.4$ indeed lead to a realistic HF abundance of $1.4 \times 10^{-8}$. To substantiate these uncertainties, we used $\mathrm{CH}$ in parallel wherever it was available. The large scatter in the ratio of $\mathrm{H}_{2}$ column densities derived from $\mathrm{HF}$ and $\mathrm{CH}$ (Table 4) reflects the underlying uncertainties. It seems likely, however, that the uncertainties in the assumed abundance of $\mathrm{CH}$ are larger. Its chemistry is more complex than that of HF (e.g., van Dishoeck \& Black 1986) and its Galactocentric abundance gradient is known to be steeper than that of oxygen (e.g., Esteban et al. 2005). The absorption of $\mathrm{CH}$ is not saturated, but the large hyperfine splitting (the components of the $536 \mathrm{GHz}$ transition are separated by $19.3 \mathrm{~km} \mathrm{~s}^{-1}$ ) and the fact that $\mathrm{CH}$ appears in emission at the velocities of the hot cores produce complex spectra. Our analysis can deal with this complexity: For the fits in Figs. 1 and 2 only one emission component with a given velocity, width, excitation temperature, and opacity had to be assumed. Derived column densities are representative for $N_{\mathrm{H}_{2}}$ (cf Liszt \& Lucas 2002). The abundances 


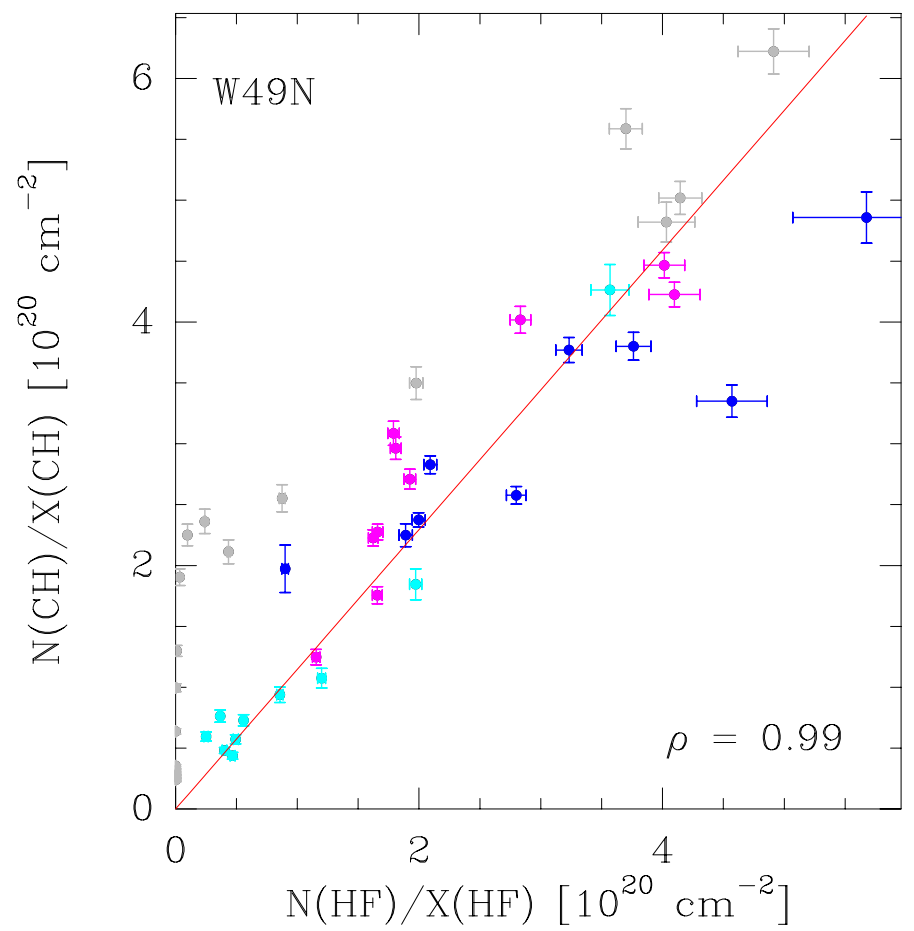

Fig. E.1. Comparison between $\mathrm{H}_{2}$ column densities derived from $\mathrm{CH}$ and from HF toward $\mathrm{W} 49 \mathrm{~N}$ in velocity intervals of $1 \mathrm{~km} \mathrm{~s}^{-1}$. The color code for the velocities is the same as in Fig. 6 (velocities above $60 \mathrm{~km} \mathrm{~s}^{-1}$ are shown in gray).

in Table 4 derived from the column densities of both $\mathrm{H}_{2}$ tracers agree reasonably well, although differences occur when HF tends to saturate.

For the example of W49N, Fig. E.1 shows a comparison between the $\mathrm{H}_{2}$ column densities derived from $\mathrm{CH}$ and those derived from HF. The correlation is statistically significant, with a false-alarm probability below $1 \%$ and with a correlation coefficient of 0.99 . The slope of the regression line exceeds the expected slope of unity by $15 \%$ and corresponds to an $\mathrm{X}(\mathrm{HF}) / \mathrm{X}(\mathrm{CH})$ ratio of 0.35 instead of the canonical value 0.4 , which is compatible with its standard deviation. Only velocities void of saturated absorption in $\mathrm{HF}$ and of emission in $\mathrm{CH}$ were considered $\left(>30 \mathrm{~km} \mathrm{~s}^{-1}\right)$. Data points with $N / \sigma_{\mathrm{N}}<5$ were discarded from this analysis. The result (slope and coefficient of the correlation) is fairly robust against relaxing these restrictions within reasonable limits.

\section{Appendix F: Cross-calibration between PACS and GREAT}

To confirm the continuum calibration in GREAT's H-channel, here we compare our flux densities with those measured by PACS. For the three targets of the OIobservations, Herschel/PACS data exist (range spectroscopy mode B3A R1, Herschel key program "PRISMAS") and were taken in pointed chop/nod mode. Figure F.1 shows a comparison between the GREAT spectra and the OIline as observed with PACS.
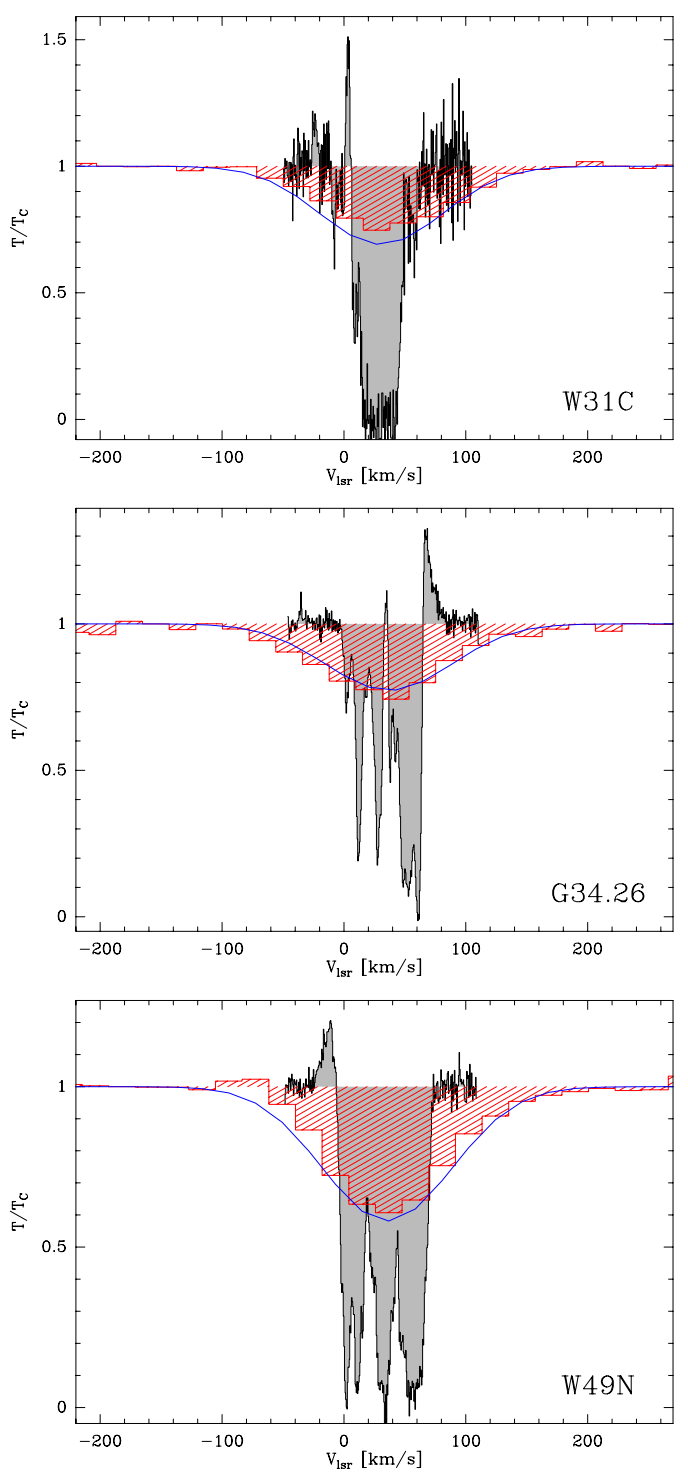

Fig. F.1. Comparison between GREAT spectra of the O Iline (gray filled) with those observed with Herschel/PACS (red hatched). The blue spectrum results from the convolution of the GREAT spectrum with a Gaussian of $86.6 \mathrm{~km} \mathrm{~s}^{-1}$ width (FWHM). All spectra are normalized to unity at the continuum level. From top to bottom: W31C (G10.62), G34.26, and W49N.

We note that the large width of the spectral response of PACS fills the saturated absorption with the continuum emission on either side of the line profile. We convolved our high-resolution data with a Gaussian profile with width $86.6 \mathrm{~km} \mathrm{~s}^{-1}$ (FWHM, from Poglitsch et al. 2010, their Table 1), assumed to represent the response of PACS to a spectrally unresolved feature. The skewness of the spectral response (PACS Observer's Manual, Version 2.5.1) was not taken into account but may explain the difference between the PACS spectra and our simulation. The continuum fluxes of PACS agree with ours (Table 5) within 4 to $14 \%$ (for G34.26 and W49N, respectively), and within $22 \%$ for W31C. 